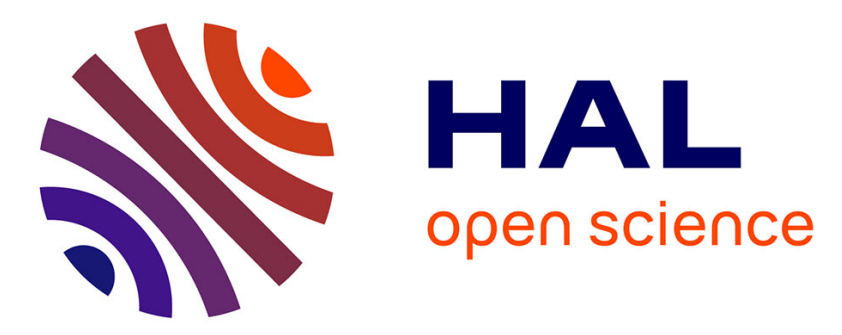

\title{
Abstract nonconforming error estimates and application to boundary penalty methods for diffusion equations and time-harmonic Maxwell's equations
}

Alexandre Ern, Jean-Luc Guermond

\section{- To cite this version:}

Alexandre Ern, Jean-Luc Guermond. Abstract nonconforming error estimates and application to boundary penalty methods for diffusion equations and time-harmonic Maxwell's equations. Computational Methods in Applied Mathematics, 2018, 18 (3), pp.451-475. 10.1515/cmam-2017-0058 . hal-01563594v4

\author{
HAL Id: hal-01563594 \\ https://hal.science/hal-01563594v4
}

Submitted on 20 Nov 2017

HAL is a multi-disciplinary open access archive for the deposit and dissemination of scientific research documents, whether they are published or not. The documents may come from teaching and research institutions in France or abroad, or from public or private research centers.
L'archive ouverte pluridisciplinaire HAL, est destinée au dépôt et à la diffusion de documents scientifiques de niveau recherche, publiés ou non, émanant des établissements d'enseignement et de recherche français ou étrangers, des laboratoires publics ou privés. 


\title{
Abstract nonconforming error estimates and application to boundary penalty methods for diffusion equations and time-harmonic Maxwell's equations
}

\author{
Alexandre Ern* \\ Jean-Luc Guermond ${ }^{\dagger}$
}

November 20, 2017

\begin{abstract}
We devise a novel framework for the error analysis of finite element approximations to low-regularity solutions in nonconforming settings where the discrete trial and test spaces are not subspaces of their exact counterparts. The key is to use face-to-cell extension operators so as to give a weak meaning to the normal or tangential trace on each mesh face individually for vector fields with minimal regularity and then to prove the consistency of this new formulation by means of some recently-derived mollification operators that commute with the usual derivative operators. We illustrate the technique on Nitsche's boundary penalty method applied to a scalar diffusion equation and to the time-harmonic Maxwell's equations. In both cases, the error estimates are robust in the case of heterogeneous material properties. We also revisit the error analysis framework proposed by Gudi where a trimming operator is introduced to map discrete test functions into conforming test functions. This technique also gives error estimates for minimal regularity solutions, but the constants depend on the material properties through contrast factors.
\end{abstract}

Keywords. Finite elements, Nonconforming methods, Error estimates, Minimum regularity, Nitsche method, Boundary penalty, Elliptic equations, Time-harmonic Maxwell equations.

\section{Introduction}

The error analysis of the finite element approximation of Partial Differential Equations (PDEs) is well understood; see, e.g., the textbooks $[7,8,13]$. The most basic result is Céa's Lemma [11] which is valid when the approximation setting is conforming (the discrete trial and test spaces are subspaces of their exact counterparts) and exactly consistent (the discrete forms are restrictions of the exact ones to the discrete spaces). Departures from this setting are usually handled in the literature by invoking Strang's Lemmas [27]. Strang's First Lemma assumes that the approximation setting is conforming but handles the case where the discrete forms differ from their exact counterpart. Strang's Second Lemma deals with nonconforming approximation settings and is frequently invoked in the literature for the error analysis of nonconforming techniques. For instance, many authors have adopted this approach to analyze discontinuous Galerkin (dG) methods (see, e.g., $[12,14]$ and the references therein).

One important shortcoming of Strang's Second Lemma is that one needs to insert the exact solution in the first argument of the discrete sesquilinear (or bilinear) form. Unfortunately, this is only possible if one assumes some additional regularity on the exact solution which often goes beyond the regularity provided by the weak formulation of the model problem at hand. For instance, when approximating a diffusion equation of the form $-\nabla \cdot(\kappa \nabla u)=f$ in some Lipschitz domain $D$ in $\mathbb{R}^{d}$, one is essentially led to assume that $\kappa \nabla u \in \boldsymbol{H}^{r}(D)$ with $r>\frac{1}{2}$ so as to make sense of the normal component $\boldsymbol{n} \cdot(\kappa \nabla u)$ at the mesh interfaces. Although this assumption is not really restrictive for the Laplace equation in a polyhedron $(\kappa \equiv 1)$, since elliptic regularity guarantees the existence of an index $r>\frac{1}{2}$ so that $u \in H^{1+r}(D)$, it becomes unrealistic in problems with discontinuous coefficients. Similarly, for the time-harmonic Maxwell's equations of the

\footnotetext{
${ }^{1}$ Université Paris-Est, CERMICS (ENPC), 77455 Marne-la-Vallée cedex 2, France and INRIA Paris, 2 rue Simone Iff, 75589 Paris, France.

${ }^{2}$ Department of Mathematics, Texas A\&M University 3368 TAMU, College Station, TX 77843, USA
} 
form $\tilde{\mu} \boldsymbol{A}+\nabla \times(\kappa \nabla \times \boldsymbol{A})=\boldsymbol{f}$ in some Lipschitz domain $D$ in $\mathbb{R}^{3}$, one is led to assume $\kappa \nabla \times \boldsymbol{A} \in \boldsymbol{H}^{r}(D)$ with $r>\frac{1}{2}$ so as to make sense of the tangential component $\boldsymbol{n} \times(\kappa \nabla \times \boldsymbol{A})$ at the mesh interfaces, but this assumption becomes unrealistic in problems with discontinuous coefficients. Let us mention in passing that we use boldface notation for $\mathbb{R}^{d}$-valued fields in $D$.

One possible way forward to overcome the limitations of Strang's Second Lemma has been proposed by Gudi [18]. The main idea is to introduce an operator that transforms the discrete test functions into elements of the exact test space. We call this operator a trimming operator, and we call the resulting error estimate a trimmed error estimate. The reason for our terminology is that one can view the elements in the kernel of the trimming operator as discrete (test) functions that are only needed to "stabilize" the bilinear form $a_{h}$, but do not contribute to the interpolatory properties of the approximation setting. We also observe that a trimming operator is one of the fundamental ingredients in the abstract setting recently devised by Veeser and Zanotti [28] to obtain quasi-optimal energy-norm error estimates for nonconforming finite element methods applied to symmetric elliptic PDEs. The trimmed error estimate in [18] (which is sometimes referred to as "medius analysis") has been applied to the Interior Penalty dG (IPDG) approximation of the Laplace equation with a source term $f \in L^{2}(D)$ (and to a fourth-order problem also in [18], to the Stokes equations in [3], and to the linear elasticity equations in [10]). In the present work, we show how to apply the trimmed error estimate to the diffusion equation with heterogeneous material property $\kappa$ and source term $f \in L^{q}(D)$ with $q \in\left(2_{*}, 2\right], 2_{*}=\frac{2 d}{2+d}$, and also to the time-harmonic Maxwell's equations with heterogeneous material properties $\widetilde{\mu}, \kappa$ and source term $\boldsymbol{f} \in \boldsymbol{L}^{2}(D)$. For simplicity, we focus for both model problems on the use of $H^{1}$-conforming finite elements combined with the boundary penalty method of Nitsche [23] to enforce weakly Dirichlet boundary conditions. The main benefit of the trimmed error analysis is that it allows one to derive error estimates as soon as the exact solution is in $\left\{v \in H^{1}(D) \mid \nabla \cdot(\kappa \nabla v) \in L^{q}(D)\right\}, q \in\left(2_{*}, 2\right]$, for the diffusion equation, and as soon as the exact solution is in $\left\{\boldsymbol{A} \in \boldsymbol{H}(\operatorname{curl} ; D) \mid \nabla \times(\kappa \nabla \times \boldsymbol{A}) \in \boldsymbol{L}^{2}(D)\right\}$ for the time-harmonic Maxwell's equations.

One difficulty still remains with the trimmed error estimate in the case of strong contrasts in the material property $\kappa$ since the error estimates feature a constant that is typically proportional to the square-root of a contrast factor associated with $\kappa$ (and, in the case of Maxwell's equations, there is also a dependency on the square-root of a local magnetic Reynolds number). These dependencies originate from the usage of the trimming operator to perform some averaging to achieve the desired conformity property, but this averaging, in turn, precludes the derivation of stability and approximation properties for the trimming operator that are local to a mesh cell. To remedy this difficulty, we devise in this work a novel approach which avoids the use of any trimming operator and instead hinges on a decomposition of the discrete sesquilinear (or bilinear) form as $a_{h}(\cdot, \cdot)=\tilde{a}_{h}(\cdot, \cdot)+s_{h}(\cdot, \cdot)$ where $\tilde{a}_{h}(\cdot, \cdot)$ is meant to ensure a consistency property and $s_{h}(\cdot, \cdot)$ is added for stabilization purposes. The crucial ingredient is then to devise a form $a_{\sharp}(\cdot, \cdot)$ with the following key properties: $a_{\sharp}\left(\cdot, w_{h}\right)$ coincides with $\tilde{a}_{h}\left(\cdot, w_{h}\right)$ for any discrete function $w_{h}$ when the first argument is discrete, $a_{\sharp}\left(\cdot, w_{h}\right)$ makes unambiguous sense when the first argument is a function with some minimal regularity, and $a_{\sharp}\left(\cdot, w_{h}\right)$ enjoys a consistency property with the right-hand side of the discrete problem. The construction of $a_{\sharp}$ is achieved by giving a meaning by duality to the normal or tangential component of vector fields at the mesh faces using face-to-cell lifting operators which we construct herein following ideas similar to those in Bernardi and Hecht [5], Amrouche et al. [1]. Since the proof of the above key consistency property hinges on some recently-devised mollification operators, we call the resulting error estimate a mollified error estimate.

In the present work, we present an abstract setting for the mollified error analysis and then we show how to apply it to Nitsche's boundary penalty method to approximate the diffusion equation and the time-harmonic Maxwell's equations. In both cases, the error estimates are robust with respect to the contrast in material properties. The mollified error analysis is applicable as soon as the exact solution is in $\left\{v \in H^{1}(D) \mid \kappa \nabla v \in \boldsymbol{L}^{p}(D), \nabla \cdot(\kappa \nabla v) \in L^{q}(D)\right\}, p>2$ and $q>\frac{2 d}{2+d}$, for the diffusion equation, and as soon as the exact solution is in $\left\{\boldsymbol{A} \in \boldsymbol{H}(\operatorname{curl} ; D) \mid \kappa \nabla \times \boldsymbol{A} \in \boldsymbol{L}^{p}(D), \nabla \times(\kappa \nabla \times \boldsymbol{A}) \in \boldsymbol{L}^{2}(D)\right\}, p>2$, for the time-harmonic Maxwell's equations. Owing to the Sobolev Embedding Theorem, the requirements that $\kappa \nabla v \in \boldsymbol{L}^{p}(D)$ or $\kappa \nabla \times \boldsymbol{A} \in \boldsymbol{L}^{p}(D), p>2$, hold true whenever $\kappa \nabla v \in \boldsymbol{H}^{r}(D)$ or $\kappa \nabla \times \boldsymbol{A} \in \boldsymbol{H}^{r}(D), r>0$, and these are minimal requirements to achieve some decay rate with respect to the mesh-size in the error estimate. Notice also that these requirements are in general compatible with the regularity pickup estimates available in the literature for the model problems at hand (see, e.g., Jochmann [20], Bonito et al. [6] for the Maxwell's equations).

This paper is organized as follows. In Section 2, we present the two model problems on which we will 
illustrate the present developments: the diffusion equation and the time-harmonic Maxwell's equations. In Section 3, we introduce the finite element setting and illustrate our abstract discrete setting on Nitsche's boundary penalty method for our two model problems. Section 4 is concerned with abstract error estimates. We first recall Strang's Lemmas, then we present Gudi's trimmed error estimate, and we finish with our novel mollified error estimate. Section 5 contains some useful analysis tools. We first recall some recent results from [15] on shrinking-based mollification operators that commute with the usual derivative operators $(\nabla$, $\nabla \times$, and $\nabla \cdot$ ). Then, we present some inverse inequalities useful for the trimmed error analysis and some extension operators that are crucial for the mollified error analysis since they allow us to give a weak meaning to the normal or tangential component of vector fields. Finally, in Section 6 and in Section 7, we show how to apply the trimmed error estimate and the mollified error estimate to our two model problems from Section 3. Although we have focused for brevity on the application to Nitsche's boundary penalty method, we do not anticipate any significant difficulty in extending the present analysis to other nonconforming approximation methods, such as Crouzeix-Raviart-type finite elements and discontinuous Galerkin (dG) methods, since in all the cases the key issue is to give a suitable weak meaning to the normal or tangential trace of vector fields with minimal regularity.

\section{Model problem}

We introduce in this section an abstract model problem and illustrate the setting on the diffusion equation and the time-harmonic Maxwell's equations.

\subsection{Abstract setting}

Let $V$ and $W$ be two Banach spaces; to stay general, we consider linear spaces over the field of complex numbers. Let $a(\cdot, \cdot)$ be a bounded sesquilinear form on $V \times W$, and let $\ell(\cdot)$ be a bounded antilinear form on $W$, i.e., $\ell \in W^{\prime}$. We consider the following abstract model problem:

$$
\left\{\begin{array}{l}
\text { Find } u \in V \text { such that } \\
a(u, w)=\ell(w), \quad \forall w \in W,
\end{array}\right.
$$

which we assume to be well-posed in the sense of Hadamard; that is to say, there is a unique solution and this solution depends continuously on the data. The well-posedness of the model problem (2.1) can be characterized by invoking Banach's Closed Range and Open Mapping Theorems; see Nečas [22] and Babuška and Aziz [2, p. 112].

Theorem 2.1 (Banach-Nečas-Babuška (BNB)). Assume that $W$ is a reflexive Banach space. The problem (2.1) is well-posed if and only if:

$$
\begin{aligned}
& \inf _{v \in V} \sup _{w \in W} \frac{|a(v, w)|}{\|v\|_{V}\|w\|_{W}}=: \alpha>0, \\
& \forall w \in W, \quad[\forall v \in V, a(v, w)=0] \Longrightarrow[w=0] .
\end{aligned}
$$

In particular, the a priori estimate $\|u\|_{V} \leq \frac{1}{\alpha}\|\ell\|_{W^{\prime}}$ holds true.

It is implicitly understood here and in what follows that the above infimum and supremum are taken over nonzero arguments.

\subsection{Diffusion equation}

To illustrate the abstract setting introduced above, let us consider a bounded Lipschitz polyhedron $D$ in $\mathbb{R}^{d}$ with $d \geq 2$. Let $f \in L^{q}(D)$ be a source term with $q \in\left(2_{*}, 2\right], 2_{*}:=\frac{2 d}{2+d}$ (so that $q \in(1,2]$ if $d=2$, and $q \in\left(\frac{6}{5}, 2\right]$ if $\left.d=3\right)$. We consider the following model problem: find $u: D \rightarrow \mathbb{R}$ s.t.

$$
-\nabla \cdot(\kappa \nabla u)=f \quad \text { in } D, \quad u=0 \quad \text { on } \partial D
$$

where $\kappa \in L^{\infty}(D)$ takes values a.e. in $D$ in the interval $\left[\kappa_{b}, \kappa_{\sharp}\right]$ with $0<\kappa_{b} \leq \kappa_{\sharp}<\infty$. 
Let us introduce the Hilbert space $H^{1}(D)=\left\{v \in L^{2}(D) \mid \nabla v \in \boldsymbol{L}^{2}(D)\right\}$ and its zero-trace subspace $H_{0}^{1}(D)=\left\{v \in H^{1}(D) \mid \gamma^{\mathrm{g}}(v)=0\right\}$ where $\gamma^{\mathrm{g}}: H^{1}(D) \rightarrow H^{\frac{1}{2}}(\partial D)$ is the well-known trace operator. To be dimensionally coherent, we equip the space $H^{1}(D)$ with the norm $\|v\|_{H^{1}(D)}=\left(\|v\|_{L^{2}(D)}^{2}+\ell_{D}^{2}\|\nabla v\|_{L^{2}(D)}^{2}\right)^{\frac{1}{2}}$, where $\ell_{D}$ is some length scale characteristic of $D$, e.g., the diameter of $D$. The model problem (2.3) fits the abstract setting of $(2.1)$ with $V=W=H_{0}^{1}(D)$ and

$$
a(v, w):=\int_{D} \kappa \nabla v \cdot \nabla w \mathrm{~d} x, \quad \ell(w):=\int_{D} f w \mathrm{~d} x,
$$

and its well-posedness follows from the Lax-Milgram Lemma. In particular, we have

$$
\begin{aligned}
|a(v, w)| & \leq \kappa_{\sharp}\|\nabla v\|_{\boldsymbol{L}^{2}(D)}\|\nabla w\|_{\boldsymbol{L}^{2}(D)}, \\
a(v, v) & \geq \kappa_{b}\|\nabla v\|_{\boldsymbol{L}^{2}(D)}^{2},
\end{aligned}
$$

for all $v, w \in H_{0}^{1}(D)$. Note that $\|v\|_{H^{1}(D)} \leq\left(1+C_{\mathrm{PS}, D}^{-2}\right)^{\frac{1}{2}} \ell_{D}\|\nabla v\|_{L^{2}(D)}$ owing to the Poincaré-Steklov inequality $C_{\mathrm{PS}, D}\|v\|_{L^{2}(D)} \leq \ell_{D}\|\nabla v\|_{L^{2}(D)}$ for all $v \in H_{0}^{1}(D)$. Note also that a Sobolev embedding implies that $w \in L^{q^{\prime}}(D)$ for all $w \in H^{1}(D)$, where $q^{\prime}$ is the conjugate number of $q$, i.e., $\frac{1}{q}+\frac{1}{q^{\prime}}=1$, so that the linear form $\ell(\cdot)$ is well-defined owing to Hölder's inequality.

Remark 2.2 (Extensions). Most of what is said in the paper generalizes when lower-order terms are added to the PDE in (2.3), $\kappa$ is tensor-valued, and non-homogeneous Dirichlet conditions are imposed.

\subsection{Time-harmonic Maxwell's equations}

As a second example to illustrate the abstract setting introduced above, we consider the time-harmonic Maxwell's equations in a bounded Lipschitz polyhedron $D$ in $\mathbb{R}^{3}$. Let $\boldsymbol{f} \in \boldsymbol{L}^{2}(D)$ be a source term. We consider the following model problem: find $\boldsymbol{A}: D \rightarrow \mathbb{R}^{3}$ s.t.

$$
\widetilde{\mu} \boldsymbol{A}+\nabla \times(\kappa \nabla \times \boldsymbol{A})=\boldsymbol{f}, \quad \boldsymbol{A}_{\mid \partial D} \times \boldsymbol{n}=\mathbf{0} .
$$

We assume that $\widetilde{\mu} \in L^{\infty}(D ; \mathbb{C}), \kappa \in B V(D ; \mathbb{C}) \cap L^{\infty}(D ; \mathbb{C})$, and we set $\mu_{\sharp}=\operatorname{ess}_{\sup _{\boldsymbol{x} \in D}}|\widetilde{\mu}(\boldsymbol{x})|$ and $\kappa_{\sharp}=$ $\operatorname{ess} \sup _{\boldsymbol{x} \in D}|\kappa(\boldsymbol{x})|$. We also assume the following positivity condition: There are real numbers $\theta, \mu_{\mathrm{b}}>0$, and $\kappa_{b}>0$ so that, letting $\mu_{r}:=\Re\left(e^{i \theta} \widetilde{\mu}\right)$ and $\kappa_{r}:=\Re\left(e^{i \theta} \kappa\right)$, we have

$$
\underset{\boldsymbol{x} \in D}{\operatorname{essinf}} \mu_{r}(\boldsymbol{x}) \geq \mu_{b} \quad \text { and } \quad \underset{\boldsymbol{x} \in D}{\operatorname{essinf}} \kappa_{r}(\boldsymbol{x}) \geq \kappa_{b} .
$$

The positivity condition (2.7) fails when the two complex numbers $\widetilde{\mu}$ and $\kappa$ are collinear and point in opposite directions. If it is the case, the model problem (2.6) is an eigenvalue problem, otherwise it is a boundary-value problem. The model problem (2.6) can be derived from the Maxwell's equations in the time-harmonic regime, i.e., under the assumption that the time variation is of the form $e^{i \omega t}$ where $\omega$ is the angular frequency and $i^{2}=-1$. One example is the Helmholtz problem where $\boldsymbol{A}$ stands for the electric field, $\widetilde{\mu}=-\omega^{2} \epsilon+i \omega \sigma$ with $\epsilon$ the electric permittivity and $\sigma$ the electric conductivity, $\kappa=\mu^{-1}$ with $\mu$ the magnetic permeability, and $\boldsymbol{f}=-i \omega \boldsymbol{j}_{s}$ with $\boldsymbol{j}_{s}$ an imposed current. Another example is the eddy-current problem where $\boldsymbol{A}$ stands for the magnetic field, $\widetilde{\mu}=i \omega \mu, \kappa=\sigma^{-1}$, and $\boldsymbol{f}=\nabla \times\left(\sigma^{-1} \boldsymbol{j}_{s}\right)$.

Let us introduce the Hilbert space $\boldsymbol{H}(\operatorname{curl} ; D)=\left\{\boldsymbol{b} \in \boldsymbol{L}^{2}(D) \mid \nabla \times \boldsymbol{b} \in \boldsymbol{L}^{2}(D)\right\}$ and its zero-trace subspace $\boldsymbol{H}_{0}(\operatorname{curl} ; D)=\left\{\boldsymbol{b} \in \boldsymbol{H}(\operatorname{curl} ; D) \mid \gamma^{\mathrm{c}}(\boldsymbol{b})=\mathbf{0}\right\}$ where $\gamma^{\mathrm{c}}: \boldsymbol{H}(\operatorname{curl} ; D) \rightarrow \boldsymbol{H}^{-\frac{1}{2}}(\partial D):=\left(\boldsymbol{H}^{\frac{1}{2}}(\partial D)\right)^{\prime}$ is the tangential trace operator s.t.

$$
\left\langle\gamma^{\mathrm{c}}(\boldsymbol{b}), \boldsymbol{l}\right\rangle_{\partial D}:=\int_{D} \boldsymbol{b} \cdot \nabla \times \boldsymbol{w}(\boldsymbol{l}) \mathrm{d} x-\int_{D}(\nabla \times \boldsymbol{b}) \cdot \boldsymbol{w}(\boldsymbol{l}) \mathrm{d} x,
$$

for all $\boldsymbol{b} \in \boldsymbol{H}(\operatorname{curl} ; D)$ and all $\boldsymbol{l} \in \boldsymbol{H}^{\frac{1}{2}}(\partial D)$ where $\boldsymbol{w}(\boldsymbol{l}) \in \boldsymbol{H}^{1}(D)$ is a lifting of $\boldsymbol{l}$ s.t. $\gamma^{\mathrm{g}}(\boldsymbol{w}(\boldsymbol{l}))=\boldsymbol{l}$ (componentwise) and $\langle\cdot, \cdot\rangle_{\partial D}$ denotes the duality pairing between $\boldsymbol{H}^{-\frac{1}{2}}(\partial D)$ and $\boldsymbol{H}^{\frac{1}{2}}(\partial D)$. Note that $\gamma^{\mathrm{c}}(\boldsymbol{b})=\boldsymbol{b}_{\mid \partial D} \times \boldsymbol{n}$ whenever the field $\boldsymbol{b}$ is smooth. To be dimensionally coherent, we equip the space $\boldsymbol{H}$ (curl; $D$ ) with the norm $\|\boldsymbol{b}\|_{\boldsymbol{H}(\operatorname{curl} ; D)}=\left(\|\boldsymbol{b}\|_{\boldsymbol{L}^{2}(D)}^{2}+\ell_{D}^{2}\|\nabla \times \boldsymbol{b}\|_{\boldsymbol{L}^{2}(D)}^{2}\right)^{\frac{1}{2}}$. The model problem (2.6) fits the abstract setting of (2.1) with $V=W=\boldsymbol{H}_{0}(\operatorname{curl} ; D)$ and

$$
a(\boldsymbol{v}, \boldsymbol{b}):=\int_{D}(\widetilde{\mu} \boldsymbol{v} \cdot \overline{\boldsymbol{b}}+\kappa \nabla \times \boldsymbol{v} \cdot \nabla \times \overline{\boldsymbol{b}}) \mathrm{d} x, \quad \ell(\boldsymbol{b}):=\int_{D} \boldsymbol{f} \cdot \overline{\boldsymbol{b}} \mathrm{d} x
$$


and its well-posedness follows from the Lax-Milgram Lemma. In particular, we have

$$
\begin{aligned}
|a(\boldsymbol{v}, \boldsymbol{b})| & \leq \max \left(\mu_{\sharp}, \ell_{D}^{-2} \kappa_{\sharp}\right)\|\boldsymbol{v}\|_{\boldsymbol{H}(\operatorname{curl} ; D)}\|\boldsymbol{b}\|_{\boldsymbol{H}(\operatorname{curl} ; D)}, \\
\Re\left(\mathrm{e}^{i \theta} a(\boldsymbol{b}, \boldsymbol{b})\right) & \geq \min \left(\mu_{b}, \ell_{D}^{-2} \kappa_{b}\right)\|\boldsymbol{b}\|_{\boldsymbol{H}(\operatorname{curl} ; D)}^{2},
\end{aligned}
$$

for all $\boldsymbol{v}, \boldsymbol{b} \in \boldsymbol{H}_{0}(\operatorname{curl} ; D)$.

Remark 2.3 (Extensions). Most of what is said in the paper generalizes when the non-homogeneous Dirichlet condition $\gamma^{\mathrm{c}}(\boldsymbol{A})=\boldsymbol{g}$ is enforced in (2.6) with $\boldsymbol{g}$ in the range of the trace map $\gamma^{\mathrm{c}}$.

\section{Discrete problem}

We now formulate a discrete version of the problem (2.1) by using the Galerkin method. The central idea in the Galerkin method consists of replacing the infinite-dimensional spaces $V$ and $W$ by finite-dimensional spaces $V_{h}$ and $W_{h}$ that are members of sequences of spaces $\left(V_{h}\right)_{h \rightarrow 0},\left(W_{h}\right)_{h \rightarrow 0}$ endowed with some approximation properties as $h \rightarrow 0$. The norms in $V_{h}$ and $W_{h}$ are denoted by $\|\cdot\|_{V_{h}}$ and $\|\cdot\|_{W_{h}}$, respectively. The discrete problem is formulated as follows:

$$
\left\{\begin{array}{l}
\text { Find } u_{h} \in V_{h} \text { such that } \\
a_{h}\left(u_{h}, w_{h}\right)=\ell_{h}\left(w_{h}\right), \quad \forall w_{h} \in W_{h}
\end{array}\right.
$$

where $a_{h}(\cdot, \cdot)$ is a bounded sesquilinear form on $V_{h} \times W_{h}$ and $\ell_{h}(\cdot)$ is a bounded antilinear form on $W_{h}$; note that $a_{h}(\cdot, \cdot)$ and $\ell_{h}(\cdot)$ possibly differ from $a(\cdot, \cdot)$ and $\ell(\cdot)$, respectively. We henceforth assume that $\operatorname{dim}\left(V_{h}\right)=\operatorname{dim}\left(W_{h}\right)$ and that

$$
\inf _{v_{h} \in V_{h}} \sup _{w_{h} \in W_{h}} \frac{\left|a_{h}\left(v_{h}, w_{h}\right)\right|}{\left\|v_{h}\right\|_{V_{h}}\left\|w_{h}\right\|_{W_{h}}}=: \alpha_{h}>0, \quad \forall h>0
$$

so that the discrete problem (3.1) is well-posed.

\subsection{Finite element setting}

Let $\left(\mathcal{T}_{h}\right)_{h>0}$ be a shape-regular sequence of meshes; we assume that each mesh covers $D$ exactly. To avoid technical questions regarding hanging nodes, we also suppose that each mesh is matching, i.e., for all cells $K, K^{\prime} \in \mathcal{T}_{h}$ such that $K \neq K^{\prime}$ and $K \cap K^{\prime} \neq \emptyset$, the set $K \cap K^{\prime}$ is a common vertex, edge, or face of both $K$ and $K^{\prime}$ (with obvious extensions in higher space dimensions). Given a mesh $\mathcal{T}_{h}$, the elements in $K \in \mathcal{T}_{h}$ are closed sets in $\mathbb{R}^{d}$ by convention, and they are all assumed to be constructed from a single reference cell $\widehat{K}$ through affine, bijective, geometric transformations $\boldsymbol{T}_{K}: \widehat{K} \rightarrow K$. For a mesh cell $K \in \mathcal{T}_{h}$, we define $\check{\mathcal{T}}_{K}$ to be the collection of the mesh cells in $\mathcal{T}_{h}$ that touch $K$, i.e., the mesh cells that share a vertex, an edge or a face (in dimension 3) with $K$, plus $K$ itself. We define $D_{K}:=\operatorname{int}\left(\cup_{K^{\prime} \in \check{\mathcal{T}}_{K}} K^{\prime}\right)$; note that the number of cells composing $\check{\mathcal{T}}_{K}$ is uniformly bounded owing to the shape-regularity of the mesh sequence.

The set of the mesh faces is denoted $\mathcal{F}_{h}$. This set is partitioned into the subset of the interfaces denoted $\mathcal{F}_{h}^{\circ}$ and the subset of the boundary faces denoted $\mathcal{F}_{h}^{\partial}$. Each interface $F$ is oriented by choosing one unit normal vector $\boldsymbol{n}_{F}$. The boundary faces are oriented by using the outward normal vector that we denote $\boldsymbol{n}$. Given an interface $F \in \mathcal{F}_{h}^{\circ}$, we denote by $K_{l}$ (left cell) and $K_{r}$ (right cell) the two cells such that $F=K_{l} \cap K_{r}$ and $\boldsymbol{n}_{F}$ points from $K_{l}$ to $K_{r}$. This convention allows us to define the notion of jump across $F$ for any smooth enough function $v$ as follows:

$$
\llbracket v \rrbracket_{F}(\boldsymbol{x}):=v_{\mid K_{l}}(\boldsymbol{x})-v_{\mid K_{r}}(\boldsymbol{x}) \quad \text { a.e. } \boldsymbol{x} \text { in } F .
$$

We consider a reference finite element in the sense of Ciarlet $\left(\widehat{K}, \widehat{P}^{\mathrm{g}}, \widehat{\Sigma}^{\mathrm{g}}\right)$. (The superscript ${ }^{\mathrm{g}}$ is intended to remind us that this finite element will be used to build a finite-dimensional subspace composed of functions whose gradient in $D$ is integrable.) We think of $\left(\widehat{K}, \widehat{P}^{\mathrm{g}}, \widehat{\Sigma}^{\mathrm{g}}\right)$ as a scalar-valued finite element with some degrees of freedom that require point evaluations, for instance $\left(\widehat{K}, \widehat{P}^{\mathrm{g}}, \widehat{\Sigma}^{\mathrm{g}}\right)$ could be a Lagrange finite element. The local shape functions are denoted $\left(\widehat{\theta}_{i}\right)_{i \in \mathcal{N}}$; recall that $\sigma_{i}\left(\widehat{\theta}_{j}\right)=\delta_{i j}$ for all $\sigma_{i} \in \widehat{\Sigma}^{\mathrm{g}}$, and all $i, j \in \mathcal{N}$. At this point, we do not need to know the exact structure of the reference element. One 
typically assumes that there exists $k \in \mathbb{N}$ such that $\mathbb{P}_{k, d} \subset \widehat{P}^{\mathrm{g}}$, where $\mathbb{P}_{k, d}$ is the vector space composed of the $d$-variate polynomials of degree at most $k$.

In order to construct $H^{1}$-conforming approximation spaces based on $\left(\mathcal{T}_{h}\right)_{h>0}$ using the above reference finite element, we introduce the pullback by the geometric map $\boldsymbol{T}_{K}$ which we denote by $\psi_{K}^{\mathrm{g}}$, i.e., $\psi_{K}^{\mathrm{g}}(v)=$ $v \circ \boldsymbol{T}_{K}$. Then we set

$$
\begin{aligned}
& P^{\mathrm{g}}\left(\mathcal{T}_{h}\right):=\left\{v_{h} \in L^{1}(D) \mid v_{h \mid K} \in P_{K}, \forall K \in \mathcal{T}_{h}, \quad \llbracket v_{h} \rrbracket_{F}=0, \forall F \in \mathcal{F}_{h}^{\circ}\right\}, \\
& P_{0}^{\mathrm{g}}\left(\mathcal{T}_{h}\right):=P^{\mathrm{g}}\left(\mathcal{T}_{h}\right) \cap H_{0}^{1}(D),
\end{aligned}
$$

where $P_{K}:=\left(\psi_{K}^{g}\right)^{-1}\left(\widehat{P}^{\mathrm{g}}\right)$. Let $\mathcal{F}_{K}$ be the collection of the faces of $K$, and for all $F \in \mathcal{F}_{K}$, let $\gamma_{K, F}$ be the corresponding trace map. For the above construction of $P^{\mathrm{g}}\left(\mathcal{T}_{h}\right)$ to be meaningful, we assume that for any mesh interface $F \in \mathcal{F}_{h}^{\circ}$ s.t. $F=K_{l} \cap K_{r}$, we have $\gamma_{K_{l}, F}\left(P_{K_{l}}\right)=\gamma_{K_{r}, F}\left(P_{K_{r}}\right)=: P_{F}$. We call $P_{F}$ the finite element trace space. For instance, if $\widehat{P}=\mathbb{P}_{k, d}$ and $\widehat{K}$ is a simplex, then $P_{F}$ is composed of the restriction of $d$-variate polynomials of degree at most $k$ to $F$.

Remark 3.1 (Reference cell). The construction of the $H^{1}$-conforming space $P^{\mathrm{g}}\left(\mathcal{T}_{h}\right)$ by means of a reference cell $\widehat{K}$ is classical in the context of finite elements. On polyhedral meshes, one can also consider $H^{1}$ conforming spaces defined locally in each cell of the mesh, as in the Virtual Element Method [4].

\subsection{Boundary penalty for the diffusion equation}

We are going to illustrate our results on the so-called boundary penalty method of Nitsche [23]. Let us first consider the diffusion equation from Section 2.2. To avoid technicalities, we assume that there is a partition of $D$ into $M$ disjoint Lipschitz polyhedra $D_{1}, \cdots, D_{M}$ so that $\kappa_{\mid D_{i}}$ is constant for all $1 \leq i \leq M$, and we assume that the meshes in $\left(\mathcal{T}_{h}\right)_{h>0}$ are fitted to this partition, so that, for all $h>0$ and all $K \in \mathcal{T}_{h}, \kappa_{\mid K}$ is constant; we use the notation $\kappa_{K}:=\kappa_{\mid K}$.

Let $V_{h}:=P^{\mathrm{g}}\left(\mathcal{T}_{h}\right)$ be the $H^{1}$-conforming finite element space based on $\mathcal{T}_{h}$ introduced in (3.4). For the diffusion equation, the discrete forms $a_{h}(\cdot, \cdot)$ and $\ell_{h}(\cdot)$ are defined by

$$
\begin{aligned}
a_{h}\left(v_{h}, w_{h}\right) & :=\int_{D} \kappa \nabla v_{h} \cdot \nabla w_{h} \mathrm{~d} x-\int_{\partial D}\left(\boldsymbol{n} \cdot \kappa \nabla v_{h}\right) w_{h} \mathrm{~d} s+\int_{\partial D} \eta_{h} v_{h} w_{h} \mathrm{~d} s \\
\ell_{h}\left(w_{h}\right) & :=\int_{D} f w_{h} \mathrm{~d} x,
\end{aligned}
$$

for all $v_{h}, w_{h} \in V_{h}$. It is useful to decompose the discrete bilinear form as $a_{h}(\cdot, \cdot)=\tilde{a}_{h}(\cdot, \cdot)+s_{h}(\cdot, \cdot)$ where

$$
\begin{aligned}
& \tilde{a}_{h}\left(v_{h}, w_{h}\right):=\int_{D} \kappa \nabla v_{h} \cdot \nabla w_{h} \mathrm{~d} x-\int_{\partial D}\left(\boldsymbol{n} \cdot \kappa \nabla v_{h}\right) w_{h} \mathrm{~d} s, \\
& s_{h}\left(v_{h}, w_{h}\right):=\int_{\partial D} \eta_{h} v_{h} w_{h} \mathrm{~d} s .
\end{aligned}
$$

The discrete bilinear form $\tilde{a}_{h}(\cdot, \cdot)$ is meant to ensure a consistency property, and the discrete bilinear form $s_{h}(\cdot, \cdot)$ is added for stabilization purposes. The penalty parameter is defined by setting $\eta_{h}:=\eta_{0} \rho_{h}$ where the user-dependent factor $\eta_{0}>0$ has yet to be chosen large enough (see Lemma 3.2 below) and where

$$
\rho_{h \mid F}:=\frac{\kappa_{K_{F}}}{h_{F}}, \quad \forall F \in \mathcal{F}_{h}^{\partial},
$$

where $K_{F}$ is the unique mesh cell having $F$ as a face.

We equip the space $V_{h}$ with the following norm:

$$
\left\|v_{h}\right\|_{V_{h}}:=\left(\left\|\kappa^{\frac{1}{2}} \nabla v_{h}\right\|_{L^{2}(D)}^{2}+\left\|\rho_{h}^{\frac{1}{2}} v_{h}\right\|_{L^{2}(\partial D)}^{2}\right)^{\frac{1}{2}}, \quad \forall v_{h} \in V_{h}
$$

Since $\left\|v_{h}\right\|_{V_{h}}=0$ implies that $v_{h}$ is constant on $D$ and vanishes on $\partial D$, and hence vanishes everywhere in $D$, we infer that $\|\cdot\|_{V_{h}}$ is indeed a norm on $V_{h}$. Furthermore, owing to the assumed shape-regularity of the 
mesh sequence, there is $c_{I}$, uniform with respect to $h$ (but depending on the shape-regularity of the mesh sequence and on the reference finite element), such that

$$
\left\|v_{h}\right\|_{L^{2}(F)} \leq c_{I} h_{F}^{-\frac{1}{2}}\left\|v_{h}\right\|_{L^{2}\left(K_{F}\right)},
$$

for all $v_{h} \in V_{h}$ and all $F \in \mathcal{F}_{h}^{\partial}$. The following stability result is classical; we simply state it without proof (see, e.g., [12, Lem. 4.12] for a proof in the context of dG methods).

Lemma 3.2 (Coercivity and well-posedness). Suppose that $\eta_{h}$ is defined by (3.7) with $\eta_{0}>\frac{1}{4} n_{\partial} c_{I}^{2}$ where $n_{\partial}$ is the maximum number of boundary faces that a mesh cell can have ( $n_{\partial} \leq d$ for simplicial meshes). Then, the following coercivity property holds true:

$$
a_{h}\left(v_{h}, v_{h}\right) \geq \alpha\left\|v_{h}\right\|_{V_{h}}^{2}, \quad \forall v_{h} \in V_{h},
$$

with $\alpha:=\frac{\eta_{0}-\frac{1}{4} n_{\partial} c_{I}^{2}}{1+\eta_{0}}$. Consequently, the discrete problem (3.1) is well-posed for the diffusion equation.

\subsection{Boundary penalty for Maxwell's equations}

Nitsche's boundary penalty method can also be applied to the time-harmonic Maxwell's equations from Section 2.3. We assume that there is a partition of $D$ into $M$ disjoint Lipschitz polyhedra $D_{1}, \cdots, D_{M}$ so that $\widetilde{\mu}_{\mid D_{i}}$ and $\kappa_{\mid D_{i}}$ are constant for all $1 \leq i \leq M$, and we assume that the meshes in $\left(\mathcal{T}_{h}\right)_{h>0}$ are fitted to this partition, so that, for all $h>0$ and all $K \in \mathcal{T}_{h}, \widetilde{\mu}_{\mid K}$ and $\kappa_{\mid K}$ are constant; we use the notation $\mu_{\sharp, K}:=\left|\widetilde{\mu}_{\mid K}\right|, \mu_{r, K}:=\mu_{r \mid K}, \kappa_{\sharp, K}:=\left|\kappa_{\mid K}\right|$, and $\kappa_{r, K}:=\kappa_{r \mid K}$, where we recall that $\mu_{r}:=\Re\left(e^{i \theta} \widetilde{\mu}\right)$ and $\kappa_{r}:=\Re\left(e^{i \theta} \kappa\right)$.

Let $\boldsymbol{V}_{h}=\boldsymbol{P}^{\mathrm{g}}\left(\mathcal{T}_{h}\right)$ be the $\boldsymbol{H}^{1}$-conforming finite element space based on $\mathcal{T}_{h}$, where $\boldsymbol{P}^{\mathrm{g}}\left(\mathcal{T}_{h}\right)$ is the vectorvalued version of the finite element space $P^{\mathrm{g}}\left(\mathcal{T}_{h}\right)$ considered above for the diffusion equation. For the time-harmonic Maxwell's equations, the discrete forms $a_{h}(\cdot, \cdot)=\tilde{a}_{h}(\cdot, \cdot)+s_{h}(\cdot, \cdot)$ and $\ell_{h}(\cdot)$ are defined by

$$
\begin{aligned}
\tilde{a}_{h}\left(\boldsymbol{v}_{h}, \boldsymbol{b}_{h}\right) & :=\int_{D}\left(\widetilde{\mu} \boldsymbol{v}_{h} \cdot \overline{\boldsymbol{b}}_{h}+\kappa \nabla \times \boldsymbol{v}_{h} \cdot \nabla \times \overline{\boldsymbol{b}}_{h}\right) \mathrm{d} x+\int_{\partial D}\left(\boldsymbol{n} \times\left(\kappa \nabla \times \boldsymbol{v}_{h}\right)\right) \cdot \overline{\boldsymbol{b}}_{h} \mathrm{~d} s, \\
s_{h}\left(\boldsymbol{v}_{h}, \boldsymbol{b}_{h}\right) & :=\int_{\partial D} \eta_{h}\left(\boldsymbol{v}_{h} \times \boldsymbol{n}\right) \cdot\left(\overline{\boldsymbol{b}}_{h} \times \boldsymbol{n}\right) \mathrm{d} s, \\
\ell_{h}\left(\boldsymbol{b}_{h}\right) & :=\int_{D} \boldsymbol{f} \cdot \overline{\boldsymbol{b}}_{h} \mathrm{~d} x,
\end{aligned}
$$

for all $\boldsymbol{v}_{h}, \boldsymbol{b}_{h} \in \boldsymbol{V}_{h}$. The discrete sesquilinear form $\tilde{a}_{h}(\cdot, \cdot)$ is meant to ensure a consistency property, and the discrete sesquilinear form $s_{h}(\cdot, \cdot)$ is added for stabilization purposes. The penalty parameter is defined by setting $\eta_{h}=\eta_{0} e^{-i \theta} \rho_{h}$ where the user-dependent factor $\eta_{0}>0$ has yet to be chosen large enough (see Lemma 3.3 below), and where

$$
\rho_{h \mid F}:=\frac{\left|\kappa_{K_{F}}\right|^{2}}{\kappa_{r, K_{F}} h_{F}}, \quad \forall F \in \mathcal{F}_{h}^{\partial},
$$

where $K_{F}$ is the unique mesh cell having $F$ as a face.

We equip the space $\boldsymbol{V}_{h}$ with the following norm:

$$
\left\|\boldsymbol{b}_{h}\right\|_{\boldsymbol{V}_{h}}:=\left(\left\|\mu_{r}^{\frac{1}{2}} \boldsymbol{b}_{h}\right\|_{\boldsymbol{L}^{2}(D)}^{2}+\left\|\kappa_{r}^{\frac{1}{2}} \nabla \times \boldsymbol{b}_{h}\right\|_{\boldsymbol{L}^{2}(D)}^{2}+\left\|\rho_{h}^{\frac{1}{2}}\left(\boldsymbol{b}_{h} \times \boldsymbol{n}\right)\right\|_{\boldsymbol{L}^{2}(\partial D)}^{2}\right)^{\frac{1}{2}}, \quad \forall \boldsymbol{b}_{h} \in \boldsymbol{V}_{h},
$$

where $\eta_{\theta h}=\cos (\theta) \eta_{h}$. The following stability result is proved using the same arguments as in the proof of Lemma 3.2.

Lemma 3.3 (Coercivity and well-posedness). Suppose that $\eta_{h}$ is defined by (3.12) with $\eta_{0}>\frac{1}{4} n_{\partial} c_{I}^{2}$. Then, the following coercivity property holds true:

$$
\Re\left(e^{i \theta} a_{h}\left(\boldsymbol{b}_{h}, \boldsymbol{b}_{h}\right)\right) \geq \alpha\left\|\boldsymbol{b}_{h}\right\|_{\boldsymbol{V}_{h}}^{2}, \quad \forall \boldsymbol{b}_{h} \in \boldsymbol{V}_{h},
$$

with $\alpha:=\frac{\eta_{0}-\frac{1}{4} n_{\partial} c_{I}^{2}}{1+\eta_{0}}$. Consequently, the discrete problem (3.1) is well-posed for the Maxwell's equations. 


\section{Abstract error estimates}

There are many ways to investigate the approximation properties of the above discrete problem (3.1). Since $u_{h}$ may not be a member of $V, u$ and $u_{h}$ may be objects of different nature. This poses the question of defining a common ground for the discrete solution $u_{h}$ and the exact solution $u$ to measure the error. For this purpose, we assume that it is meaningful to define the linear space

$$
V_{b}:=V+V_{h}
$$

We equip the space $V_{b}$ with a norm denoted $\|\cdot\|_{V_{b}}$ which we assume extends the discrete norm $\|\cdot\|_{V_{h}}$ to $V_{b}$, i.e., there exists a real number $c_{b}$ so that

$$
\left\|v_{h}\right\|_{V_{b}} \leq c_{b}\left\|v_{h}\right\|_{V_{h}}, \quad \forall v_{h} \in V_{h} .
$$

The goal of this section is to bound the error $u-u_{h}$ using the $\|\cdot\|_{V_{b}}$-norm. Note that even in the conforming case where $V_{b}$ and $V$ coincide as linear spaces, choosing $\|\cdot\|_{V_{b}}$ to be different from $\|\cdot\|_{V}$ can be useful for the error analysis.

\subsection{A basic error identity}

Our starting point is the following (relatively straightforward) error identity. Recall that the norm of any antilinear form $\phi_{h} \in W_{h}^{\prime}:=\mathcal{L}\left(W_{h} ; \mathbb{C}\right)$, is defined by $\left\|\phi_{h}\right\|_{W_{h}^{\prime}}:=\sup _{w_{h} \in W_{h}} \frac{\left|\phi_{h}\left(w_{h}\right)\right|}{\left\|w_{h}\right\|_{W_{h}}}$.

Lemma 4.1 (Error identity). Assume that the discrete inf-sup condition (3.2) is satisfied. Then, the following identity holds true:

$$
\left\|u-u_{h}\right\|_{V_{\mathrm{b}}}=\inf _{v_{h} \in V_{h}}\left[\left\|u-v_{h}\right\|_{V_{\mathrm{b}}}+\frac{c_{\mathrm{b}}}{\alpha_{h}}\left\|\delta_{h}\left(v_{h}\right)\right\|_{W_{h}^{\prime}}\right],
$$

where $\delta_{h}: V_{h} \rightarrow W_{h}^{\prime}$, which we call consistency error, is defined by

$$
\left\langle\delta_{h}\left(v_{h}\right), w_{h}\right\rangle_{W_{h}^{\prime}, W_{h}}:=\ell_{h}\left(w_{h}\right)-a_{h}\left(v_{h}, w_{h}\right) .
$$

Proof. Let $v_{h} \in V_{h}$. The triangle inequality, (4.2), stability, and the fact that $a_{h}\left(u_{h}, w_{h}\right)=\ell_{h}\left(w_{h}\right)$ for all $w_{h} \in W_{h}$ imply that

$$
\begin{aligned}
\left\|u-u_{h}\right\|_{V_{\mathrm{b}}} & \leq\left\|u-v_{h}\right\|_{V_{\mathrm{b}}}+\left\|u_{h}-v_{h}\right\|_{V_{\mathrm{b}}} \leq\left\|u-v_{h}\right\|_{V_{\mathrm{b}}}+c_{\mathrm{b}}\left\|u_{h}-v_{h}\right\|_{V_{h}} \\
& \leq\left\|u-v_{h}\right\|_{V_{\mathrm{b}}}+\frac{c_{\mathrm{b}}}{\alpha_{h}} \sup _{w_{h} \in W_{h}} \frac{\left|a_{h}\left(u_{h}-v_{h}, w_{h}\right)\right|}{\left\|w_{h}\right\|_{W_{h}}} \\
& =\left\|u-v_{h}\right\|_{V_{\mathrm{b}}}+\frac{c_{b}}{\alpha_{h}} \sup _{w_{h} \in W_{h}} \frac{\left|\left\langle\delta_{h}\left(v_{h}\right), w_{h}\right\rangle_{W_{h}^{\prime}, W_{h}}\right|}{\left\|w_{h}\right\|_{W_{h}}} .
\end{aligned}
$$

Since $v_{h}$ is arbitrary in $V_{h}$ and recalling the definition of the norm of the discrete antilinear form $\delta_{h}\left(v_{h}\right)$, we conclude that $\left\|u-u_{h}\right\|_{V_{b}} \leq r_{h}$, where $r_{h}$ denotes the right-hand side of (4.3). Finally, taking $v_{h}=u_{h}$ in the infimum and observing that $\delta_{h}\left(u_{h}\right)$ vanishes identically on $W_{h}$, we infer that $\left\|u-u_{h}\right\|_{V_{b}}=r_{h}$.

\subsection{Strang's Lemmas}

The traditional form of Strang's First Lemma consists of assuming that the approximation setting is conforming; that is to say, $V_{h} \subset V$ and $W_{h} \subset W$. This implies that the linear spaces $V$ and $V_{b}$ coincide; however, these spaces may be equipped with different norms.

Lemma 4.2 (Strang 1). Assume the following: (i) $V_{h} \subset V$ and $W_{h} \subset W$; (ii) The sesquilinear form a $(\cdot, \cdot)$ is bounded on $V_{b} \times W_{h}$ with norm

$$
\|a\|_{V_{\mathrm{b}}, W_{h}}:=\sup _{v \in V_{\mathrm{b}}} \sup _{w_{h} \in W_{h}} \frac{\left|a\left(v, w_{h}\right)\right|}{\|v\|_{V_{\mathrm{b}}}\left\|w_{h}\right\|_{W_{h}}} .
$$


Then, the following error estimate holds true:

$$
\left\|u-u_{h}\right\|_{V_{b}} \leq \inf _{v_{h} \in V_{h}}\left[\left(1+c_{b} \frac{\|a\|_{V_{b}, W_{h}}}{\alpha_{h}}\right)\left\|u-v_{h}\right\|_{V_{b}}+\frac{c_{b}}{\alpha_{h}}\left\|\delta_{h}^{\mathrm{St1}}\right\|_{W_{h}^{\prime}}\right],
$$

with $\delta_{h}^{\mathrm{st1}}: V_{h} \rightarrow W_{h}^{\prime}$ defined by

$$
\left\langle\delta_{h}^{\mathrm{St1}}\left(v_{h}\right), w_{h}\right\rangle_{W_{h}^{\prime}, W_{h}}:=\ell_{h}\left(w_{h}\right)-\ell\left(w_{h}\right)+a\left(v_{h}, w_{h}\right)-a_{h}\left(v_{h}, w_{h}\right) .
$$

Proof. This is an easy consequence of the error identity (4.3) after one has observed that

$$
\ell_{h}\left(w_{h}\right)-a_{h}\left(v_{h}, w_{h}\right)=\ell_{h}\left(w_{h}\right)-\ell\left(w_{h}\right)+a\left(u, w_{h}\right)+\left[a\left(v_{h}, w_{h}\right)-a\left(v_{h}, w_{h}\right)\right]-a_{h}\left(v_{h}, w_{h}\right),
$$

since $a\left(u, w_{h}\right)=\ell\left(w_{h}\right)$ for all $w_{h} \in W_{h} \subset W$. One concludes by invoking the boundedness of $a$ on $V_{\mathrm{b}} \times W_{h}$.

The main inconvenient of the above estimate is that is assumes that the discrete setting is conforming. This shortcoming is traditionally addressed in the literature by invoking Strang's Second Lemma where one supposes that the discrete sesquilinear form $a_{h}(\cdot, \cdot)$ can be extended as a bounded sesquilinear form $a_{b}(\cdot, \cdot)$ on $V_{b} \times W_{h}$.

Lemma 4.3 (Strang 2). Assume that the discrete sesquilinear form $a_{h}(\cdot, \cdot)$ admits a bounded extension $a_{b}(\cdot, \cdot)$ on $V_{b} \times W_{h}$ with norm

$$
\left\|a_{b}\right\|_{V_{b}, W_{h}}:=\sup _{v \in V_{b}} \sup _{w_{h} \in W_{h}} \frac{\left|a_{b}\left(v, w_{h}\right)\right|}{\|v\|_{V_{b}}\left\|w_{h}\right\|_{W_{h}}} .
$$

Then, the following error estimate holds true:

$$
\left\|u-u_{h}\right\|_{V_{b}} \leq\left(1+c_{b} \frac{\left\|a_{b}\right\|_{V_{b}, W_{h}}}{\alpha_{h}}\right) \inf _{v_{h} \in V_{h}}\left\|u-v_{h}\right\|_{V_{b}}+\frac{c_{b}}{\alpha_{h}}\left\|\ell_{h}-a_{b}(u, \cdot)\right\|_{W_{h}^{\prime}} .
$$

Proof. This is also an easy consequence of the error identity (4.3) after one writes

$$
\ell_{h}\left(w_{h}\right)-a_{h}\left(v_{h}, w_{h}\right)=\ell_{h}\left(w_{h}\right)+\left[a_{b}\left(u, w_{h}\right)-a_{b}\left(u, w_{h}\right)\right]-a_{b}\left(v_{h}, w_{h}\right),
$$

and uses the boundedness of $a_{b}$ on $V_{b} \times W_{h}$.

The key problem with the above estimate is that, in general, it is not possible to extend $a_{h}(\cdot, \cdot)$ to $V_{b} \times W_{h}$ unless one requires some regularity assumption on the exact solution. For instance, for the boundary penalty method, this requirement is $\kappa \nabla u \in \boldsymbol{H}^{r}(D)$ with $r>\frac{1}{2}$ in the case of the diffusion equation, and it is $\kappa \nabla \times \boldsymbol{A} \in \boldsymbol{H}^{r}(D)$ with $r>\frac{1}{2}$ in the case of the Maxwell equations. These requirements are unrealistic if the model coefficients are nonsmooth.

\subsection{Alternative error estimates}

In this section, we present two alternative error estimates that avoid extending the discrete sesquilinear form $a_{h}(\cdot, \cdot)$ to $V_{b} \times W_{h}$. We still need a regularity assumption on the exact solution, but this assumption is milder than that required to extend $a_{h}(\cdot, \cdot)$. To stay general, we formalize this regularity assumption by assuming that $u \in V_{\mathrm{S}}$ where $V_{\mathrm{S}}$ is a dense subspace of $V$. We set

$$
V_{\sharp}:=V_{\mathrm{S}}+V_{h},
$$

and we note that $V_{\sharp}$ is a subspace of $V_{b}$. We equip the space $V_{\sharp}$ with a norm $\|\cdot\|_{V_{\sharp}}$ that we suppose to be (slightly) stronger than the norm $\|\cdot\|_{V_{b}}$ restricted to $V_{\sharp}$; specifically, we assume that

$$
\|v\|_{V_{b}} \leq c_{b}\|v\|_{V_{\sharp}}, \quad \forall v \in V_{\sharp} .
$$

We use the same constant $c_{b}$ in (4.11) and in (4.2) to simplify the notation; we could consider two constants and call $c_{b}$ the largest of the two. We refer the reader to $\S 6.2$ and $\S 7.2$ where examples for the spaces $V_{\mathrm{S}}$ and $V_{\sharp}$ and the corresponding norms are given. Our starting point is the following result where we do not separate the notions of consistency and boundedness by triangle inequalities. 
Lemma 4.4 (Key error estimate). Assume that the exact solution $u$ is in $V_{\mathrm{S}}$. Assume the following consistency/boundedness property: There is a real number $\omega_{\sharp h}$ so that

$$
\left\|\delta_{h}\left(v_{h}\right)\right\|_{W_{h}^{\prime}} \leq \omega_{\sharp h}\left\|u-v_{h}\right\|_{V_{\sharp}}, \quad \forall v_{h} \in V_{h},
$$

with $\delta_{h}: V_{h} \rightarrow W_{h}^{\prime}$ defined by (4.4). Then, the following holds true:

$$
\left\|u-u_{h}\right\|_{V_{\mathrm{b}}} \leq c_{b}\left(1+\frac{\omega_{\sharp h}}{\alpha_{h}}\right) \inf _{v_{h} \in V_{h}}\left\|u-v_{h}\right\|_{V_{\sharp}} .
$$

Moreover, if the following bound holds true for some real number $c_{\sharp}$ uniform with respect to $h$ :

$$
\left\|v_{h}\right\|_{V_{\sharp}} \leq c_{\sharp}\left\|v_{h}\right\|_{V_{h}}, \quad \forall v_{h} \in V_{h},
$$

then we have the quasi-optimal error estimate

$$
\left\|u-u_{h}\right\|_{V_{\sharp}} \leq\left(1+c_{\sharp} \frac{\omega_{\sharp h}}{\alpha_{h}}\right) \inf _{v_{h} \in V_{h}}\left\|u-v_{h}\right\|_{V_{\sharp}} .
$$

Proof. The error estimate (4.13) is a direct consequence of Lemma 4.1 and consistency/boundedness. For the proof of (4.15), we proceed as in the proof of Lemma 4.1, and we write

$$
\begin{aligned}
\left\|u-u_{h}\right\|_{V_{\sharp}} & \leq\left\|u-v_{h}\right\|_{V_{\sharp}}+\left\|v_{h}-u_{h}\right\|_{V_{\sharp}} \\
& \leq\left\|u-v_{h}\right\|_{V_{\sharp}}+c_{\sharp}\left\|v_{h}-u_{h}\right\|_{V_{h}} \\
& \leq\left\|u-v_{h}\right\|_{V_{\sharp}}+\frac{c_{\sharp}}{\alpha_{h}} \sup _{w_{h} \in W_{h}} \frac{\left|a_{h}\left(u_{h}-v_{h}, w_{h}\right)\right|}{\left\|w_{h}\right\|_{W_{h}}} \\
& =\left\|u-v_{h}\right\|_{V_{\sharp}}+\frac{c_{\sharp}}{\alpha_{h}}\left\|\delta_{h}\left(v_{h}\right)\right\|_{W_{h}^{\prime}} \leq\left\|u-v_{h}\right\|_{V_{\sharp}}+\frac{c_{\sharp} \omega_{\sharp h}}{\alpha_{h}}\left\|u-v_{h}\right\|_{V_{\sharp}},
\end{aligned}
$$

and we conclude by taking the infimum over $v_{h} \in V_{h}$.

Remark 4.5 (Quasi-optimality). The error estimate (4.15) is said to be quasi-optimal since the same norm is used to measure the error and the best-approximation error of the solution in $V_{h}$.

\subsubsection{Trimmed error estimate}

One possible way forward to overcome the limitations of Strang's Second Lemma has been proposed by Gudi [18]. The key idea is to introduce a so-called trimming operator $T: W_{h} \rightarrow W \cap W_{h}$ that transforms the discrete test functions into (discrete) objects that are conforming in $W$.

Lemma 4.6 (Trimmed error estimate). Assume that the exact solution $u$ is in $V_{\mathrm{s}}$. Consider any map $T: W_{h} \rightarrow W \cap W_{h}$ such that the following properties hold true: (i) There exists a real number $\omega_{V_{\sharp}, W_{h}}^{\text {tri }}$ so that

$$
\left\|a(u, T(\cdot))-a_{h}\left(v_{h}, T(\cdot)\right)\right\|_{W_{h}^{\prime}} \leq \omega_{V_{\sharp}, W_{h}}^{\mathrm{tri}}\left\|u-v_{h}\right\|_{V_{\sharp}}, \quad \forall v_{h} \in V_{h} ;
$$

(ii) There exists a real number $\varpi_{V_{\sharp}, W_{h}}^{\text {tri }}$ so that

$$
\left\|\ell_{h}-\ell \circ T-a_{h}\left(v_{h},(I-T)(\cdot)\right)\right\|_{W_{h}^{\prime}} \leq \varpi_{V_{\sharp}, W_{h}}^{\mathrm{tri}}\left\|u-v_{h}\right\|_{V_{\sharp}}, \quad \forall v_{h} \in V_{h},
$$

where $I$ is the identity operator in $W_{h}$. Then, the following error estimate holds true:

$$
\left\|u-u_{h}\right\|_{V_{\mathrm{b}}} \leq c_{b}\left(1+\frac{\omega_{V_{\sharp}, W_{h}}^{\mathrm{tri}}+\varpi_{V_{\sharp}, W_{h}}^{\mathrm{tri}}}{\alpha_{h}}\right) \inf _{v_{h} \in V_{h}}\left\|u-v_{h}\right\|_{V_{\sharp}} .
$$

Moreover, if the discrete norm equivalence (4.14) holds true, we have the quasi-optimal error estimate

$$
\left\|u-u_{h}\right\|_{V_{\sharp}} \leq\left(1+c_{\sharp} \frac{\omega_{V_{\sharp}, W_{h}}^{\mathrm{tri}}+\varpi_{V_{\sharp}, W_{h}}^{\mathrm{tri}}}{\alpha_{h}} \inf _{v_{h} \in V_{h}}\left\|u-v_{h}\right\|_{V_{\sharp}} .\right.
$$


Proof. We observe that, for all $v_{h} \in V_{h}$ and all $w_{h} \in W_{h}$, we have

$$
\ell_{h}\left(w_{h}\right)-a_{h}\left(v_{h}, w_{h}\right)=\ell_{h}\left(w_{h}\right)-\ell\left(T\left(w_{h}\right)\right)+a\left(u, T\left(w_{h}\right)\right)-\left[a_{h}\left(v_{h}, T\left(w_{h}\right)\right)-a_{h}\left(v_{h}, T\left(w_{h}\right)\right)\right]-a_{h}\left(v_{h}, w_{h}\right)
$$

since $a\left(u, T\left(w_{h}\right)\right)=\ell\left(T\left(w_{h}\right)\right)$ for all $w_{h} \in W_{h}$. Owing to the properties (4.16) and (4.17), we infer that the consistency/boundedness property (4.12) holds true with $\omega_{\sharp h}=\omega_{V_{\sharp}, W_{h}}^{\text {tri }}+\varpi_{V_{\sharp}, W_{h}}^{\text {tri }}$. The assertions then follow from the key error estimates of Lemma 4.4 .

Remark 4.7 (Conforming case). Whenever $W_{h} \subset W$, one can take $T$ to be the canonical injection $W_{h} \hookrightarrow$ $W$. In this case, the abstract error estimate (4.18) differs from that derived in Strang's First Lemma. The reason for this is that we have exploited additional boundedness properties to derive (4.18).

\subsubsection{Mollified error estimate}

Although the trimmed error estimate presented in the previous section can overcome some shortcomings encountered with the use of Strang's Lemmas, as illustrated by the examples in Section 6 and in Section 7 , we will also see that some difficulties remain. In particular, it is not always easy to construct a trimming operator in the context of Maxwell's equations when one does not use edge elements and the faces of the domain $D$ are not orthogonal to one of the coordinate axes. Moreover, it is not simple to construct a trimming operator that exhibits suitable stability properties that are robust in the case of highly-contrasted coefficients. The goal of this section is to present a new approach for the error analysis that attempts to remedy these difficulties.

Lemma 4.8 (Mollified error estimate). Assume that the exact solution $u$ is in $V_{\mathrm{S}}$. Recall the decomposition $a_{h}(\cdot, \cdot)=\tilde{a}_{h}(\cdot, \cdot)+s_{h}(\cdot, \cdot)$. Assume that there is a sesquilinear form $a_{\sharp}(\cdot, \cdot)$ on $V_{\sharp} \times W_{h}$ that is bounded on $V_{\sharp} \times W_{h}$, i.e.,

$$
\left\|a_{\sharp}(v, \cdot)\right\|_{W_{h}^{\prime}} \leq \omega_{V_{\sharp}, W_{h}}^{\operatorname{mol}}\|v\|_{V_{\sharp}}, \quad \forall v \in V_{\sharp},
$$

and such that the following two identities hold true:

$$
\begin{array}{ll}
a_{\sharp}\left(v_{h}, w_{h}\right)=\tilde{a}_{h}\left(v_{h}, w_{h}\right) & \forall\left(v_{h}, w_{h}\right) \in V_{h} \times W_{h}, \\
a_{\sharp}\left(u, w_{h}\right)=\ell_{h}\left(w_{h}\right) & \forall w_{h} \in W_{h} .
\end{array}
$$

Assume moreover that there exists a real number $\sigma_{V_{h}, W_{h}}$ so that

$$
\left\|s_{h}\left(v_{h}, \cdot\right)\right\|_{W_{h}^{\prime}} \leq \sigma_{V_{h}, W_{h}}\left\|v-v_{h}\right\|_{V_{\sharp}}, \quad \forall v_{h} \in V_{h}, \forall v \in V .
$$

Then, the following error estimate holds true:

$$
\left\|u-u_{h}\right\|_{V_{\mathrm{b}}} \leq c_{b}\left(1+\frac{\omega_{V_{\sharp}, W_{h}}^{\mathrm{mol}}+\sigma_{V_{h}, W_{h}}}{\alpha_{h}}\right) \inf _{v_{h} \in V_{h}}\left\|u-v_{h}\right\|_{V_{\sharp}} .
$$

Moreover, if the discrete norm equivalence (4.14) holds true, we have the quasi-optimal error estimate

$$
\left\|u-u_{h}\right\|_{V_{\sharp}} \leq\left(1+c_{\sharp} \frac{\omega_{V_{\sharp}, W_{h}}^{\mathrm{mol}}+\sigma_{V_{h}, W_{h}}}{\alpha_{h}}\right) \inf _{v_{h} \in V_{h}}\left\|u-v_{h}\right\|_{V_{\sharp}} .
$$

Proof. We observe that, for all $v_{h} \in V_{h}$ and all $w_{h} \in W_{h}$, we have

$$
\left\langle\delta_{h}\left(v_{h}\right), w_{h}\right\rangle_{W_{h}^{\prime}, W_{h}}=a_{\sharp}\left(u-v_{h}, w_{h}\right)-s_{h}\left(v_{h}, w_{h}\right),
$$

where we used (4.21a)-(4.21b). Invoking now (4.20) and (4.22), we infer that the consistency/boundedness property (4.12) holds true with $\omega_{\sharp h}=\omega_{V_{\sharp}, W_{h}}^{\text {mol }}+\sigma_{V_{h}, W_{h}}$. The assertions then follow from the key error estimates of Lemma 4.4.

Remark 4.9 (Terminology). We call the estimates from Lemma 4.8 mollified error estimates since the proof of $(4.21 \mathrm{~b})$ hinges on the use of suitable mollification operators; we refer the reader to the examples presented in Section 6.2 and in Section 7.2. 


\section{Analysis tools}

We introduce in this section some analysis tools that are useful to realize the above program. These tools include commuting mollification operators in Section 5.1, inverse inequalities on faces in Section 5.2, and the localization of weak traces to faces in Section 5.3. The results of Section 5.2 are useful in the context of the trimmed error estimates, and the results of Section 5.1 and of Section 5.3 are useful in the context of the mollified error estimates. The results from Section 5.2 and Section 5.3 invoke the shape-regularity of the mesh sequence. They can be extended to polyhedral mesh sequences admitting a simplicial submesh that belongs to a shape-regular sequence in the usual sense and such that all the polyhedral cells and polygonal faces are covered by a finite number of tetrahedra and triangles with uniformly the same size.

\subsection{Mollification operators}

Smoothing by mollification (i.e., by convolution with a smooth kernel) is an important tool for the analysis and approximation of PDEs that has been introduced by Leray [21, p. 206], Sobolev [26, p. 487], and Friedrichs [17, p. 136-139]. The goal of this section is to define mollification operators that commute with the usual differential operators, and that converge optimally when the function to be smoothed is defined over a Lipschitz domain $D$ in $\mathbb{R}^{d}$. We use the shrinking technique of $D$ in [15] to avoid the need to extend the function to be smoothed outside $D$.

The starting point is to observe that Proposition 2.3 in Hofmann et al. [19] implies the existence of a vector field $j \in C^{\infty}\left(\mathbb{R}^{d}\right)$ that is globally transversal on $\partial D$ (i.e., there is a real number $\gamma>0$ such that $\boldsymbol{n}(\boldsymbol{x}) \cdot \boldsymbol{j}(\boldsymbol{x}) \geq \gamma$ at a.e. point $\boldsymbol{x}$ on $\partial D$ where $\boldsymbol{n}$ is the unit normal vector pointing outward $D)$ and $\|\boldsymbol{j}(\boldsymbol{x})\|_{\ell^{2}}=1$ for all $\boldsymbol{x} \in \partial D$. Then, one can show the following: (i) The map

$$
\boldsymbol{\varphi}_{\delta}: \mathbb{R}^{d} \ni \boldsymbol{x} \longmapsto \boldsymbol{x}-\delta \boldsymbol{j}(\boldsymbol{x}) \in \mathbb{R}^{d} .
$$

is in $C^{\infty}\left(\mathbb{R}^{d}\right)$ for all $\delta \in[0,1]$; (ii) For all $k \in \mathbb{N}$, there is $c$ such that $\max _{\boldsymbol{x} \in D}\left\|D^{k} \boldsymbol{\varphi}_{\delta}(\boldsymbol{x})-D^{k} \boldsymbol{x}\right\|_{\ell^{2}} \leq c \ell_{D}^{-k} \delta$, for all $\delta \in[0,1]$; (iii) There is $r>0$ so that

$$
\boldsymbol{\varphi}_{\delta}(D)+B(\mathbf{0}, \delta r) \subset D, \quad \forall \delta \in[0,1] .
$$

Let us consider the following kernel:

$$
\rho(\boldsymbol{y})= \begin{cases}\eta \exp \left(-\frac{1}{1-\|\boldsymbol{y}\|_{\ell^{2}}^{2}}\right), & \text { if }\|\boldsymbol{y}\|_{\ell^{2}}<1 \\ 0, & \text { if }\|\boldsymbol{y}\|_{\ell^{2}} \geq 1\end{cases}
$$

where $\eta$ is chosen so that $\int_{\mathbb{R}^{d}} \rho(\boldsymbol{y}) \mathrm{d} y=\int_{B(\mathbf{0}, 1)} \rho(\boldsymbol{y}) \mathrm{d} y=1$. Let $\delta \in[0,1]$ and let $f \in L^{1}\left(D ; \mathbb{R}^{q}\right)$ with $q=1$ if we consider scalar-valued functions and $q=d$ if we consider vector-valued functions.

We define a mollification operator as follows:

$$
\mathcal{K}_{\delta}(f)(\boldsymbol{x}):=\int_{B(\mathbf{0}, 1)} \rho(\boldsymbol{y}) \mathbb{K}_{\delta}(\boldsymbol{x}) f\left(\boldsymbol{\varphi}_{\delta}(\boldsymbol{x})+(\delta r) \boldsymbol{y}\right) \mathrm{d} y, \quad \forall \boldsymbol{x} \in D,
$$

where $\mathbb{K}_{\delta}: D \rightarrow \mathbb{R}^{q \times q}$ is a smooth field. Note that the definition (5.4) makes sense owing to (5.2). The examples we have in mind for the field $\mathbb{K}_{\delta}$ (inspired by Schöberl [24, 25]) are $\mathbb{K}_{\delta}^{\mathrm{g}}(\boldsymbol{x})=1(q=1)$, $\mathbb{K}_{\delta}^{\mathrm{c}}(\boldsymbol{x})=\mathbb{J}_{\delta}^{\mathrm{T}}(\boldsymbol{x})(q=d=3), \mathbb{K}_{\delta}^{\mathrm{d}}(\boldsymbol{x})=\operatorname{det}\left(\mathbb{J}_{\delta}(\boldsymbol{x})\right) \mathbb{J}_{\delta}^{-1}(\boldsymbol{x})(q=d)$, and $\mathbb{K}_{\delta}^{\mathrm{b}}(\boldsymbol{x})=\operatorname{det}\left(\mathbb{J}_{\delta}(\boldsymbol{x})\right)(q=1)$, where $\mathbb{J}_{\delta}$ is the Jacobian matrix of $\boldsymbol{\varphi}_{\delta}$ at $\boldsymbol{x} \in D$. The mollification operator built using the field $\mathbb{K}_{\delta}^{\mathrm{x}}$ is denoted $\mathcal{K}_{\delta}^{\mathrm{x}}$ with $\mathrm{x} \in\{\mathrm{g}, \mathrm{c}, \mathrm{d}, \mathrm{b}\}$. In what follows, we just state the main properties of the mollification operator $\mathcal{K}_{\delta}$ (where we omit the superscript if the context is unambiguous), and we refer the reader to [15] for proofs.

Lemma 5.1 (Smoothness). For all $f \in L^{1}\left(D ; \mathbb{R}^{q}\right)$ and all $\delta \in(0,1], \mathcal{K}_{\delta}(f) \in C^{\infty}\left(\bar{D} ; \mathbb{R}^{q}\right)$, i.e., $\mathcal{K}_{\delta}(f) \in$ $C^{\infty}\left(D ; \mathbb{R}^{q}\right)$ and $\mathcal{K}_{\delta}(f)$ as well as all its derivatives admit a continuous extension to $\bar{D}$.

Let $p \in[1, \infty]$. Let us set $Z^{\mathrm{g}, p}(D)=W^{1, p}(D)=\left\{f \in L^{p}(D) \mid \nabla f \in \boldsymbol{L}^{p}(D)\right\}, \boldsymbol{Z}^{\mathrm{c}, p}(D)=\{\boldsymbol{g} \in$ $\left.\boldsymbol{L}^{p}(D) \mid \nabla \times \boldsymbol{g} \in \boldsymbol{L}^{p}(D)\right\}\left(\right.$ for $d=3$ ), and $\boldsymbol{Z}^{\mathrm{d}, p}(D)=\left\{\boldsymbol{g} \in \boldsymbol{L}^{p}(D) \mid \nabla \cdot \boldsymbol{g} \in L^{p}(D)\right\}$.

Lemma 5.2 (Commuting). The following holds true:

(i) $\nabla \mathcal{K}_{\delta}^{\mathrm{g}}(f)=\mathcal{K}_{\delta}^{\mathrm{c}}(\nabla f)$, for all $f \in Z^{\mathrm{g}, p}(D)$; 
(ii) $\nabla \times \mathcal{K}_{\delta}^{\mathrm{c}}(\boldsymbol{g})=\mathcal{K}_{\delta}^{\mathrm{d}}(\nabla \times \boldsymbol{g})$, for all $\boldsymbol{g} \in \boldsymbol{Z}^{\mathrm{c}, p}(D)($ for $d=3)$;

(iii) $\nabla \cdot \mathcal{K}_{\delta}^{\mathrm{d}}(\boldsymbol{g})=\mathcal{K}_{\delta}^{\mathrm{b}}(\nabla \cdot \boldsymbol{g})$, for all $\boldsymbol{g} \in \boldsymbol{Z}^{\mathrm{d}, p}(D)$;

that is to say, the following diagrams commute:

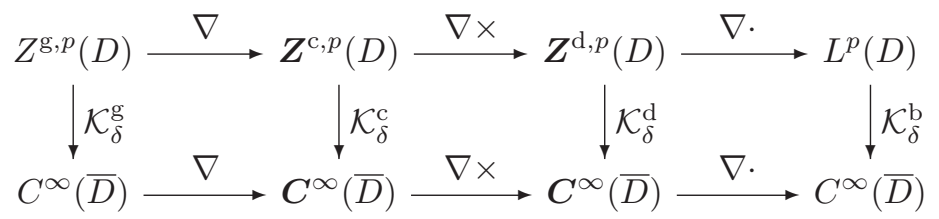

Theorem 5.3 (Convergence). The following statements hold true:

(i) There are $c, \delta_{0}>0$, uniform, such that $\left\|\mathcal{K}_{\delta}(f)\right\|_{L^{p}\left(D ; \mathbb{R}^{q}\right)} \leq c\|f\|_{L^{p}\left(D ; \mathbb{R}^{q}\right)}$ for all $f \in L^{p}\left(D ; \mathbb{R}^{q}\right)$, all $\delta \in\left[0, \delta_{0}\right]$, and all $p \in[1, \infty]$. Moreover,

$$
\lim _{\delta \rightarrow 0}\left\|\mathcal{K}_{\delta}(f)-f\right\|_{L^{p}\left(D ; \mathbb{R}^{q}\right)}=0, \quad \forall f \in L^{p}\left(D ; \mathbb{R}^{q}\right), \quad \forall p \in[1, \infty),
$$

(ii) There is $c$, uniform, such that for all $f \in W^{s, p}\left(D ; \mathbb{R}^{q}\right)$, all $\delta \in\left[0, \delta_{0}\right]$, all $s \in(0,1]$, and all $p \in[1, \infty)$ $(p \in[1, \infty]$ if $s=1)$,

$$
\left\|\mathcal{K}_{\delta}(f)-f\right\|_{L^{p}(D ; \mathbb{R} q)} \leq c \ell_{D}^{-s} \delta^{s}\|f\|_{W^{s, p}\left(D ; \mathbb{R}^{q}\right)} .
$$

Corollary 5.4 (Convergence of derivatives). The following statements hold true:

(i) $\lim _{\delta \rightarrow 0}\left\|\nabla\left(\mathcal{K}_{\delta}^{\mathrm{g}}(f)-f\right)\right\|_{\boldsymbol{L}^{p}(D)}=0, \forall f \in Z^{\mathrm{g}, p}(D)$, and if $\nabla f \in \boldsymbol{W}^{s, p}(D),\left\|\nabla\left(\mathcal{K}_{\delta}^{\mathrm{g}}(f)-f\right)\right\|_{\boldsymbol{L}^{p}(D)} \leq$ $c \ell_{D}^{-s} \delta^{s}\|\nabla f\|_{W^{s, p}(D)}$

(ii) $\lim _{\delta \rightarrow 0}\left\|\nabla \times\left(\mathcal{K}_{\delta}^{\mathrm{c}}(\boldsymbol{g})-\boldsymbol{g}\right)\right\|_{\boldsymbol{L}^{p}(D)}=0, \forall \boldsymbol{g} \in \boldsymbol{Z}^{\mathrm{c}, p}(D)$, and if $\nabla \times \boldsymbol{g} \in \boldsymbol{W}^{s, p}(D),\left\|\nabla \times\left(\mathcal{K}_{\delta}^{\mathrm{c}}(\boldsymbol{g})-\boldsymbol{g}\right)\right\|_{\boldsymbol{L}^{p}(D)} \leq$ $c \ell_{D}^{-s} \delta^{s}\|\nabla \times \boldsymbol{g}\|_{\boldsymbol{W}^{s, p}(D)}$

(iii) $\lim _{\delta \rightarrow 0}\left\|\nabla \cdot\left(\mathcal{K}_{\delta}^{\mathrm{d}}(\boldsymbol{g})-\boldsymbol{g}\right)\right\|_{L^{p}(D)}=0, \forall \boldsymbol{g} \in \boldsymbol{Z}^{\mathrm{d}, p}(D)$, and if $\nabla \cdot \boldsymbol{g} \in W^{s, p}(D),\left\|\nabla \cdot\left(\mathcal{K}_{\delta}^{\mathrm{d}}(\boldsymbol{g})-\boldsymbol{g}\right)\right\|_{L^{p}(D)} \leq$ $c \ell_{D}^{-s} \delta^{s}\|\nabla \cdot \boldsymbol{g}\|_{W^{s, p}(D)}$.

In the above statements, convergence holds true for all $p \in[1, \infty)$, and convergence rates hold true with $c$ uniform for all $\delta \in\left[0, \delta_{0}\right]$, all $s \in(0,1]$, and all $p \in[1, \infty)(p \in[1, \infty]$ if $s=1)$.

Remark 5.5 (Convergence in D). Corollary 5.4(i) strengthens the original result by Friedrichs where strong convergence of the gradient only occurs in compact subsets of $D$ (see, e.g., [9, Thm. 9.2]). Note though that Corollary 5.4(i) is valid for Lipschitz domains, whereas the original result by Friedrichs is valid for any open set.

Remark 5.6 (Density). Lemma 5.1, together with Lemma 5.2 and (5.5), implies that $C^{\infty}\left(\bar{D} ; \mathbb{R}^{q}\right)$ is dense in $Z^{\mathrm{x}, p}(D)$ for all $\mathrm{x} \in\{\mathrm{g}, \mathrm{c}, \mathrm{d}\}$.

\subsection{Inverse inequalities on faces}

Let $F \in \mathcal{F}_{h}^{\circ}$ be an interface and let $P_{F}$ be the finite element trace space defined in Section 3.1. In the following statement, we use a local length scale $\tilde{h}_{F}$ which is uniformly equivalent to the diameter $h_{F}$ of $F$ owing to the shape-regularity of the mesh sequence; the reason for this distinction is to provide a somewhat more precise geometric characterization of the relevant local length scale.

Lemma 5.7 (Verfürth's inverse inequality). Let $F \in \mathcal{F}_{h}^{\circ}$ and let $P_{F}$ be the finite element trace space. Let $\Phi_{F}: L^{2}(F) \rightarrow H^{-1}\left(D_{F}\right)$ be the map s.t. $\Phi_{F}(r)(\varphi):=\int_{F} r \varphi \mathrm{d} s$ for all $\varphi \in H_{0}^{1}\left(D_{F}\right)$ and all $r \in L^{2}(F)$, where $D_{F}$ is the interior of the set of the points of the two cells sharing $F$. Let $\tilde{h}_{F}:=\frac{\left|D_{F}\right|}{|F|}$ and let $p \in(1, \infty)$. Then there exists a constant $c$, uniform with respect to $h$ but depending on the shape-regularity of the mesh sequence and on the reference finite element, such that

$$
\|g\|_{L^{2}(F)} \leq c \tilde{h}_{F}^{-\frac{1}{2}+d\left(\frac{1}{2}-\frac{1}{p}\right)}\left\|\Phi_{F}(g)\right\|_{W_{0}^{1, p^{\prime}}\left(D_{F}\right)^{\prime}}, \quad \forall g \in P_{F},
$$

where $p^{\prime}$ is the conjugate number of $p$ (i.e., $\left.\frac{1}{p}+\frac{1}{p^{\prime}}=1\right)$ and the dual space $W_{0}^{1, p^{\prime}}\left(D_{F}\right)^{\prime}$ is equipped with the norm $\left\|\Phi_{F}(g)\right\|_{W_{0}^{1, p^{\prime}}\left(D_{F}\right)^{\prime}}=\sup _{\varphi \in W_{0}^{1, p^{\prime}}\left(D_{F}\right) ;\|\nabla \varphi\|_{L^{p^{\prime}}\left(D_{F}\right)}=1}\left|\Phi_{F}(g)(\varphi)\right|$. 
Proof. The proof hinges on the use of suitable bubble functions introduced by Verfürth and on inverse inequalities proved by mapping to the reference cell $\widehat{K}$; see $[29, \S 3.6]$ for the proof with $p=2$. The adaptation for $p \neq 2$ is straightforward and is omitted for brevity.

\subsection{Localization of weak traces to faces}

The goal of this section is to give a weak meaning to the (normal or tangential) trace of some field satisfying some minimal regularity requirements on a given mesh cell $K \in \mathcal{T}_{h}$. The key point is that the trace is given a meaning on each face of $K$ independently, and not just on the whole boundary of $K$. Let $K \in \mathcal{T}_{h}$ be a mesh cell and let $F \in \mathcal{F}_{K}$ be a face of $K$.

Let $p$ and $q$ be two real numbers such that

$$
p>2 \quad q>\frac{2 d}{2+d} .
$$

Note that $q>1$ since $d \geq 2$. Let $p^{\prime}$ be the conjugate number of $p$, i.e., $\frac{1}{p}+\frac{1}{p^{\prime}}=1$ so that $p^{\prime} \in(1,2)$. Since $x \rightarrow \frac{x d}{x+d}$ is an increasing function, there is $\widetilde{p} \in(2, p]$ such that $q \geq \frac{\widetilde{p} d}{\tilde{p}+d}$; notice that these two conditions are equivalent to $2<\widetilde{p} \leq p$ and $\frac{1}{q^{\prime}} \geq \frac{1}{\widetilde{p}^{\prime}}-\frac{1}{d}$, where $q^{\prime}$ is the conjugate number of $q$ and $\widetilde{p}^{\prime}$ that of $\widetilde{p}$.

Let us start by considering the normal component of fields defined in $K$. With the above real numbers $p, q$, and $\tilde{p}$ in hand, we consider the functional spaces

$$
\begin{aligned}
\boldsymbol{V}^{\mathrm{d}}(K) & :=\left\{\boldsymbol{v} \in \boldsymbol{L}^{p}(K) \mid \nabla \cdot \boldsymbol{v} \in L^{q}(K)\right\} \\
Y^{\mathrm{d}}(F) & :=W^{\frac{1}{\tilde{p}}, \widetilde{p}^{\prime}}(F)
\end{aligned}
$$

where the superscript refers to the fact that the normal trace is related to the divergence operator.

Lemma 5.8 (Lifting operator). There exists a constant c, uniform with respect to $h$ (but depending on the shape-regularity of the mesh sequence and on the reference finite element) and a lifting operator $E_{F}^{K}$ : $Y^{\mathrm{d}}(F) \rightarrow W^{1, \widetilde{p}^{\prime}}(K)$ such that the following holds true for any $\phi \in Y^{\mathrm{d}}(F): E_{F}^{K}(\phi)_{\mid \partial K \backslash F}=0, E_{F}^{K}(\phi)_{\mid F}=\phi$, and

$$
\left|E_{F}^{K}(\phi)\right|_{W^{1, p^{\prime}}(K)}+h_{K}^{-1+d\left(\frac{1}{q}-\frac{1}{p}\right)}\left\|E_{F}^{K}(\phi)\right\|_{L^{q^{\prime}}(K)} \leq c h_{K}^{-\frac{1}{\tilde{p}}+d\left(\frac{1}{\tilde{p}}-\frac{1}{p}\right)}\|\phi\|_{Y^{\mathrm{d}}(F)},
$$

for all $\phi \in Y^{\mathrm{d}}(F)$, with the norm $\|\phi\|_{Y^{\mathrm{d}}(F)}=\|\phi\|_{L^{\tilde{p}^{\prime}(F)}}+h_{F}^{\frac{1}{\tilde{p}}}|\phi|_{W^{\frac{1}{\tilde{p}}, \tilde{p}^{\prime}}(F)}$.

Proof. Following the ideas in, e.g., [1, Lem. 4.7] (see also [5, Cor 3.3] for similar lifting operators in a Hilbert setting), the lifting operator $E_{F}^{K}$ is constructed from a reference lifting operator $E_{\widehat{F}}^{\widehat{K}}$ where $\widehat{K}$ is the reference cell, $\widehat{F}=\left(\boldsymbol{T}_{K}\right)^{-1}(F)$, and $\boldsymbol{T}_{K}: \widehat{K} \rightarrow K$ the geometric map. The reference lifting operator is constructed by composing the zero-extension from $\widehat{F}$ to $\partial \widehat{K}$ with a bounded right-inverse of the trace map from $W^{1, \widetilde{p}^{\prime}}(\widehat{K})$ to $W^{\frac{1}{\tilde{p}}, \widetilde{p}^{\prime}}(\partial \widehat{K})$. This construction is possible since the function equal to 1 on $\widehat{F}$ and 0 on $\partial \widehat{K} \backslash \widehat{F}$ is in $W^{\frac{1}{\tilde{p}}, \widetilde{p}^{\prime}}(\partial \widehat{K})$ because $\frac{\widetilde{p}^{\prime}}{\widetilde{p}}=\frac{1}{\widetilde{p}-1}<1$. The stability bound (5.10) follows from the transformation of Sobolev norms by pullbacks associated with the geometric maps on shape-regular mesh sequences and the fact that on the reference cell $\widehat{K}$, we have $|\widehat{\psi}|_{W^{1, p^{\prime}(\widehat{K})}}+\|\widehat{\psi}\|_{L^{q^{\prime}(\widehat{K})}} \leq \widehat{c}\|\widehat{\psi}\|_{W^{1, \widetilde{p}^{\prime}(\widehat{K})}}$ since $\widetilde{p}^{\prime} \geq p^{\prime}$ and $\frac{1}{q^{\prime}} \geq \frac{1}{\widetilde{p}^{\prime}}-\frac{1}{d}$.

With the lifting operator $E_{F}^{K}$ in hand, we can define the normal component of any field $\boldsymbol{v} \in \boldsymbol{V}^{\mathrm{d}}(K)$ on the face $F$ of $K$ to be the linear form $\left(\boldsymbol{v} \cdot \boldsymbol{n}_{K}\right)_{\mid F} \in Y^{\mathrm{d}}(F)^{\prime}$ such that

$$
\left\langle\left(\boldsymbol{v} \cdot \boldsymbol{n}_{K}\right)_{\mid F}, \phi\right\rangle:=\int_{K}\left(\boldsymbol{v} \cdot \nabla E_{F}^{K}(\phi)+(\nabla \cdot \boldsymbol{v}) E_{F}^{K}(\phi)\right) \mathrm{d} x,
$$

for all $\phi \in Y^{\mathrm{d}}(F)$, where $\langle\cdot, \cdot\rangle$ denotes the duality pairing between $Y^{\mathrm{d}}(F)^{\prime}$ and $Y^{\mathrm{d}}(F)$. Note that the right-hand side of (5.11) is well-defined owing to Hölder's inequality and (5.10). 
Lemma 5.9 (Bound on normal component). There exists a constant $c$, uniform with respect to $h$ (but depending on the shape-regularity of the mesh sequence and on the reference finite element), so that the following estimate holds true for all $\boldsymbol{v} \in \boldsymbol{V}^{\mathrm{d}}(K)$,

$$
\left\|\left(\boldsymbol{v} \cdot \boldsymbol{n}_{K}\right)_{\mid F}\right\|_{Y^{\mathrm{d}}(F)^{\prime}} \leq c h_{K}^{-\frac{1}{\tilde{p}}+d\left(\frac{1}{\tilde{p}}-\frac{1}{p}\right)}\left(\|\boldsymbol{v}\|_{\boldsymbol{L}^{p}(K)}+h_{K}^{1+d\left(\frac{1}{p}-\frac{1}{q}\right)}\|\nabla \cdot \boldsymbol{v}\|_{L^{q}(K)}\right) .
$$

Moreover, we have

$$
\left|\left\langle\left(\boldsymbol{v} \cdot \boldsymbol{n}_{K}\right)_{\mid F}, \phi_{h}\right\rangle\right| \leq c\left(h_{K_{F}}^{d\left(\frac{1}{2}-\frac{1}{p}\right)}\|\boldsymbol{v}\|_{L^{p}\left(K_{F}\right)}+h_{K_{F}}^{1+d\left(\frac{1}{2}-\frac{1}{q}\right)}\|\nabla \cdot \boldsymbol{v}\|_{L^{q}\left(K_{F}\right)}\right) \times h_{F}^{-\frac{1}{2}}\left\|\phi_{h}\right\|_{L^{2}(F)},
$$

for all $\phi_{h} \in P_{F}$ and all $F \in \mathcal{F}_{h}^{\partial}$ where $K_{F}$ is the unique mesh cell having $F$ as a face.

Proof. The bound (5.12) is a direct consequence of (5.11), Hölder's inequality, and Lemma 5.8. Moreover, the bound (5.13) follows from (5.12), the following inverse inequality on $P_{F}$ :

$$
\left\|\phi_{h}\right\|_{Y^{\mathrm{d}}(F)} \leq c h_{F}^{(d-1)\left(\frac{1}{2}-\frac{1}{\tilde{p}}\right)}\left\|\phi_{h}\right\|_{L^{2}(F)}
$$

(note that $\frac{1}{2}-\frac{1}{\widetilde{p}}=\frac{1}{\widetilde{p}^{\prime}}-\frac{1}{2}$ ) and the shape-regularity of the mesh sequence.

Similar arguments can be deployed to define the tangential trace of vectors fields on a face of $K$. More specifically, let the real numbers $p, q$, and $\widetilde{p}$ be as above, and consider the functional spaces

$$
\begin{aligned}
& \boldsymbol{V}^{\mathrm{c}}(K):=\left\{\boldsymbol{v} \in \boldsymbol{L}^{p}(K) \mid \nabla \times \boldsymbol{v} \in \boldsymbol{L}^{q}(K)\right\}, \\
& \boldsymbol{Y}^{\mathrm{c}}(F):=\left\{\boldsymbol{\phi} \in \boldsymbol{W}^{\frac{1}{\tilde{p}}, \widetilde{p}^{\prime}}(F) \mid \boldsymbol{\phi} \cdot \boldsymbol{n}_{F}=0\right\},
\end{aligned}
$$

where the superscript refers to the fact that the tangential trace is related to the curl operator.

Lemma 5.10 (Lifting operator). There exist a constant $c$, uniform with respect to $h$ (but depending on the shape-regularity of the mesh sequence and on the reference finite element) and a lifting operator $E_{F}^{K}$ : $\boldsymbol{Y}^{\mathrm{c}}(F) \rightarrow \boldsymbol{W}^{1, \widetilde{p}^{\prime}}(K)$ such that the following holds true for any $\boldsymbol{\phi} \in \boldsymbol{Y}^{\mathrm{c}}(F): E_{F}^{K}(\boldsymbol{\phi})_{\mid \partial K \backslash F}=\mathbf{0}, E_{F}^{K}(\boldsymbol{\phi})_{\mid F}=\boldsymbol{\phi}$, and

$$
\left|E_{F}^{K}(\phi)\right|_{\boldsymbol{W}^{1, p^{\prime}}(K)}+h_{K}^{-1+d\left(\frac{1}{q}-\frac{1}{p}\right)}\left\|E_{F}^{K}(\boldsymbol{\phi})\right\|_{\boldsymbol{L}^{q^{\prime}}(K)} \leq c h_{K}^{-\frac{1}{\tilde{p}}+d\left(\frac{1}{\widetilde{p}}-\frac{1}{p}\right)}\|\boldsymbol{\phi}\|_{\boldsymbol{Y}^{\mathrm{c}}(F)},
$$

with the norm $\|\phi\|_{\boldsymbol{Y}^{\mathrm{c}}(F)}=\|\phi\|_{\boldsymbol{L}^{\tilde{p}^{\prime}(F)}}+h_{F}^{\frac{1}{\hat{p}}}|\phi|_{\boldsymbol{W}^{\frac{1}{\tilde{p}}, \widetilde{p}^{\prime}}(F)}$.

With this lifting operator in hand, we can define the tangential component of any field $\boldsymbol{v} \in \boldsymbol{V}^{\mathrm{c}}(K)$ on the face $F$ of $K$ to be the antilinear form $\left(\boldsymbol{v} \times \boldsymbol{n}_{K}\right)_{\mid F} \in \boldsymbol{Y}^{\mathrm{c}}(F)^{\prime}$ such that

$$
\left\langle\left(\boldsymbol{v} \times \boldsymbol{n}_{K}\right)_{\mid F}, \boldsymbol{\phi}\right\rangle:=\int_{K}\left(\boldsymbol{v} \cdot \nabla \times E_{F}^{K}(\overline{\boldsymbol{\phi}})-(\nabla \times \boldsymbol{v}) \cdot E_{F}^{K}(\overline{\boldsymbol{\phi}})\right) \mathrm{d} x,
$$

for all $\phi \in \boldsymbol{Y}^{\mathrm{c}}(F)$, where $\langle\cdot, \cdot\rangle$ denotes the duality pairing between $\boldsymbol{Y}^{\mathrm{c}}(F)^{\prime}$ and $\boldsymbol{Y}^{\mathrm{c}}(F)$. Note that the right-hand side of (5.16) is well-defined owing to Hölder's inequality and (5.15).

Lemma 5.11 (Bound on tangential component). There exists a constant c, uniform with respect to $h$ (but depending on the shape-regularity of the mesh sequence and on the reference finite element), so that the following estimate holds true for all $\boldsymbol{v} \in \boldsymbol{V}^{\mathrm{c}}(K)$,

$$
\left\|\left(\boldsymbol{v} \times \boldsymbol{n}_{K}\right)_{\mid F}\right\|_{Y^{\mathrm{c}}(F)^{\prime}} \leq c h_{K}^{-\frac{1}{\tilde{p}}+d\left(\frac{1}{\tilde{p}}-\frac{1}{p}\right)}\left(\|\boldsymbol{v}\|_{\boldsymbol{L}^{p}(K)}+h_{K}^{1+d\left(\frac{1}{p}-\frac{1}{q}\right)}\|\nabla \times \boldsymbol{v}\|_{\boldsymbol{L}^{q}(K)}\right) .
$$

Moreover, we have

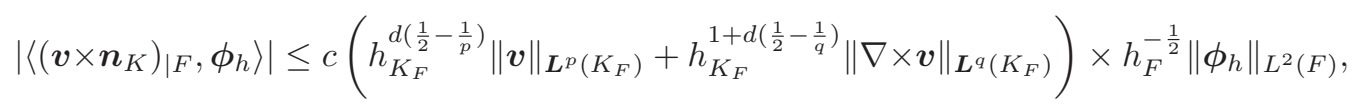

for all $\phi_{h} \in \boldsymbol{P}_{F}$ s.t. $\boldsymbol{\phi} \cdot \boldsymbol{n}_{F}=0$ and all $F \in \mathcal{F}_{h}^{\partial}$ where $K_{F}$ is the unique mesh cell having $F$ as a face. 


\section{Application to the diffusion equation}

In this section, we show how the trimmed error estimate from Lemma 4.6 and the mollified error estimate from Lemma 4.8 can be applied to the approximation of the diffusion equation using the boundary penalty method described in Section 3.2. The discrete spaces are $W_{h}=V_{h}=P^{\mathrm{g}}\left(\mathcal{T}_{h}\right)$, and the space $V_{b}:=H_{0}^{1}(D)+V_{h}$ is equipped with the norm $\|\cdot\|_{V_{b}}$ that extends to $V_{b}$ the norm $\|\cdot\|_{V_{h}}$ originally defined by (3.8) on $V_{h}$. The discrete forms $a_{h}(\cdot, \cdot)$ and $\ell_{h}(\cdot)$ are defined by (3.5). The constants in the error estimates derived in this section depend on the shape-regularity of the mesh sequence and on the reference finite element.

\subsection{Trimmed error estimate}

We define the trimming operator $T: P^{\mathrm{g}}\left(\mathcal{T}_{h}\right) \rightarrow P_{0}^{\mathrm{g}}\left(\mathcal{T}_{h}\right)$ as follows. For all $w_{h} \in P^{\mathrm{g}}\left(\mathcal{T}_{h}\right), T\left(w_{h}\right)_{\mid K}$ is defined, for all $K \in \mathcal{T}_{h}$, by zeroing out all the degrees of freedom of $w_{h}$ that are attached to vertices, edges, and faces located at the boundary $\partial D$. This type of construction has been recently analyzed in [16] in the more general context of quasi-interpolation operators in canonical finite element spaces with prescribed boundary conditions. Let $\mathcal{T}_{h}^{\partial}$ be the collection of the mesh cells touching the boundary; note that $w_{h}-T\left(w_{h}\right)$ vanishes on all the mesh cells in $\mathcal{T}_{h} \backslash \mathcal{T}_{h}^{\partial}$ but does not on the mesh cells in $\mathcal{T}_{h}^{\partial}$. For all $K \in \mathcal{T}_{h}^{\partial}$, one can prove that the following bounds hold true for all $w_{h} \in P^{\mathrm{g}}\left(\mathcal{T}_{h}\right)$ with $c$ uniform w.r.t. $h$ : If $\partial K \cap \partial D$ is composed of one or more boundary faces, then

$$
h_{K}\left\|\nabla\left(w_{h}-T\left(w_{h}\right)\right)\right\|_{L^{2}(K)}+\left\|w_{h}-T\left(w_{h}\right)\right\|_{L^{2}(K)} \leq c h_{K}^{\frac{1}{2}}\left\|w_{h}\right\|_{L^{2}(\partial K \cap \partial D)},
$$

whereas if $\partial K \cap \partial D$ is a manifold of dimension $d^{\prime}<d-1$, then

$$
h_{K}\left\|\nabla\left(w_{h}-T\left(w_{h}\right)\right)\right\|_{L^{2}(K)}+\left\|w_{h}-T\left(w_{h}\right)\right\|_{L^{2}(K)} \leq c h_{K}^{\frac{1}{2}}\left\|w_{h}\right\|_{L^{2}(F)}, \quad \forall F \in \mathcal{F}_{K}^{\partial},
$$

where $\mathcal{F}_{K}^{\partial}:=\left\{F \in \mathcal{F}_{h}^{\partial} \mid \partial K \cap \partial D \subseteq F\right\}$ is the collection of the boundary faces containing the manifold $\partial K \cap \partial D$. We introduce the contrast factor

$$
\xi_{\kappa}:=\max _{K \in \mathcal{T}_{h}^{\partial}} \frac{\kappa_{K}}{\max _{F \in \mathcal{F}_{K}^{\partial}} \kappa_{K_{F}}}
$$

where we recall that, for all $F \in \mathcal{F}_{K}^{\partial} \subset \mathcal{F}_{h}^{\partial}, K_{F}$ is the unique mesh cell having $F$ as a boundary face. Finally, let us set

$$
V_{\mathrm{S}}:=\left\{v \in H_{0}^{1}(D) \mid \nabla \cdot(\kappa \nabla v) \in L^{q}(D)\right\}
$$

with $q \in\left(2_{*}, 2\right], 2_{*}=\frac{2 d}{2+d}$, and let us equip the space $V_{\sharp}:=V_{\mathrm{S}}+V_{h}$ with the norm

$$
\|v\|_{V_{\sharp}}:=\left(\|v\|_{V_{\mathrm{b}}}^{2}+\sum_{K \in \mathcal{T}_{h}^{\partial}} \kappa_{K}^{-1} h_{K}^{2+2 d\left(\frac{1}{2}-\frac{1}{q}\right)}\|\nabla \cdot(\kappa \nabla v)\|_{L^{q}(K)}^{2}\right)^{\frac{1}{2}} .
$$

Lemma 6.1 (Trimmed error estimate). The assumptions of Lemma 4.6 hold true with the trimming operator $T: P^{\mathrm{g}}\left(\mathcal{T}_{h}\right) \rightarrow P_{0}^{\mathrm{g}}\left(\mathcal{T}_{h}\right)$ defined above, where the constants $\omega_{V_{\sharp}, V_{h}}^{\mathrm{tri}}$ and $\varpi_{V_{\sharp}, V_{h}}^{\mathrm{tri}}$ are proportional to $\xi_{\kappa}^{\frac{1}{2}}$ with the contrast factor $\xi_{\kappa}$ defined by (6.3).

Proof. (1) Let us verify that (4.16) holds true. Let $\left(v_{h}, w_{h}\right) \in V_{h} \times V_{h}$. Since $T\left(w_{h}\right) \in H_{0}^{1}(D)$, we infer that

$$
a\left(u, T\left(w_{h}\right)\right)-a_{h}\left(v_{h}, T\left(w_{h}\right)\right)=\int_{D} \kappa \nabla\left(u-v_{h}\right) \cdot \nabla T\left(w_{h}\right) \mathrm{d} x \leq\left\|u-v_{h}\right\|_{V_{\mathrm{b}}}\left\|\kappa^{\frac{1}{2}} \nabla T\left(w_{h}\right)\right\|_{\boldsymbol{L}^{2}(D)} .
$$

Since $\left\|u-v_{h}\right\|_{V_{b}} \leq\left\|u-v_{h}\right\|_{V_{\sharp}}$, we just have to prove that $\left\|\kappa^{\frac{1}{2}} \nabla T\left(w_{h}\right)\right\|_{L^{2}(D)} \leq c\left\|w_{h}\right\|_{V_{h}}$. We have $T\left(w_{h}\right)=w_{h}$ on all $K \in \mathcal{T}_{h} \backslash \mathcal{T}_{h}^{\partial}$ so that we only need to bound $\left\|\kappa^{\frac{1}{2}} \nabla T\left(w_{h}\right)\right\|_{L^{2}(K)}$ for all $K \in \mathcal{T}_{h}^{\partial}$. In this case, the triangle inequality implies that

$$
\left\|\kappa^{\frac{1}{2}} \nabla T\left(w_{h}\right)\right\|_{L^{2}(K)} \leq\left\|\kappa^{\frac{1}{2}} \nabla w_{h}\right\|_{L^{2}(K)}+\kappa_{K}^{\frac{1}{2}}\left\|\nabla \hat{w}_{h}\right\|_{L^{2}(K)}
$$


where we have set $\hat{w}_{h}:=w_{h}-T\left(w_{h}\right)$. If $\partial K \cap \partial D$ is composed of one or more boundary faces, we use the approximation property (6.1) together with the shape-regularity of the mesh sequence to infer that

$$
\kappa_{K}^{\frac{1}{2}}\left\|\nabla \hat{w}_{h}\right\|_{L^{2}(K)} \leq c \kappa_{K}^{\frac{1}{2}} h_{K}^{-\frac{1}{2}}\left\|w_{h}\right\|_{L^{2}(\partial K \cap \partial D)} \leq c^{\prime}\left(\sum_{\substack{F \in \mathcal{F}_{h}^{\partial} \\ F \subseteq \partial K \cap \partial D}}\left\|\rho_{h}^{\frac{1}{2}} w_{h}\right\|_{L^{2}(F)}^{2}\right)^{\frac{1}{2}} .
$$

Instead, if $\partial K \cap \partial D$ is a manifold of dimension $d^{\prime}<d-1$, we use the approximation property (6.2) together with the shape-regularity of the mesh sequence to infer that, for all $F \in \mathcal{F}_{K}^{\partial}$,

$$
\kappa_{K}^{\frac{1}{2}}\left\|\nabla \hat{w}_{h}\right\|_{L^{2}(K)} \leq c\left(\frac{\kappa_{K}}{\kappa_{K_{F}}}\right)^{\frac{1}{2}} \kappa_{K_{F}}^{\frac{1}{2}} h_{F}^{-\frac{1}{2}}\left\|w_{h}\right\|_{L^{2}(F)} \leq c^{\prime}\left(\frac{\kappa_{K}}{\kappa_{K_{F}}}\right)^{\frac{1}{2}}\left\|\rho_{h}^{\frac{1}{2}} w_{h}\right\|_{L^{2}(F)} .
$$

It is at this point that the contrast factor $\xi_{\kappa}$ comes into play. The reason is that $K$ is not connected to $\partial D$ by any of its faces, and (6.2) gives an estimate of $\left\|\nabla \hat{w}_{h}\right\|_{L^{2}(K)}$ that involves a boundary face $F$ that cannot be a face of $K$. There is necessarily a mismatch between $\kappa_{K}$ and the coefficient $\kappa_{K_{F}}$ involved in (3.7). We now take a boundary face in $\mathcal{F}_{K}^{\partial}$, say $F_{*}$, s.t. $\kappa_{K_{F}}$ is maximal so as to make the above upper bound as small as possible. We obtain

$$
\kappa_{K}^{\frac{1}{2}}\left\|\nabla \hat{w}_{h}\right\|_{L^{2}(K)} \leq c^{\prime}\left(\frac{\kappa_{K}}{\max _{F \in \mathcal{F}_{K}^{\partial}} \kappa_{K_{F}}}\right)^{\frac{1}{2}}\left\|\rho_{h}^{\frac{1}{2}} w_{h}\right\|_{L^{2}\left(F_{*}\right)} \leq c^{\prime}\left(\max _{K \in \mathcal{T}_{h}^{\partial}} \frac{\kappa_{K}}{\max _{F \in \mathcal{F}_{K}^{\partial}} \kappa_{K_{F}}}\right)^{\frac{1}{2}}\left\|\rho_{h}^{\frac{1}{2}} w_{h}\right\|_{L^{2}\left(F_{*}\right)} .
$$

Recalling the definition (6.3) of the contrast factor $\xi_{\kappa}$, we infer that $\kappa_{K}^{\frac{1}{2}}\left\|\nabla \hat{w}_{h}\right\|_{L^{2}(K)} \leq c^{\prime} \xi_{\kappa}^{\frac{1}{2}}\left\|\rho_{h}^{\frac{1}{2}} w_{h}\right\|_{L^{2}\left(F_{*}\right)}$ with $F_{*} \in \mathcal{F}_{K}^{\partial} \subset \mathcal{F}_{h}^{\partial}$. It is now straightforward to complete the proof of (4.16).

(2) Let us verify (4.17). Let $\left(v_{h}, w_{h}\right) \in V_{h} \times V_{h}$ and let us set $e_{h}:=u-v_{h}$ and (as above) $\hat{w}_{h}:=w_{h}-T\left(w_{h}\right)$. A direct calculation shows that

$$
\begin{aligned}
& \ell_{h}\left(w_{h}\right)-\ell\left(T\left(w_{h}\right)\right)-a_{h}\left(v_{h},(I-T)\left(w_{h}\right)\right) \\
& =\int_{D} f \hat{w}_{h} \mathrm{~d} x-\int_{D} \kappa \nabla v_{h} \cdot \nabla \hat{w}_{h} \mathrm{~d} x+\int_{\partial D}\left(\boldsymbol{n} \cdot \kappa \nabla v_{h}\right) \hat{w}_{h} \mathrm{~d} s-\int_{\partial D} \eta_{h} v_{h} \hat{w}_{h} \mathrm{~d} s \\
& =\sum_{K \in \mathcal{T}_{h}^{\partial}} \int_{K}-\nabla \cdot\left(\kappa \nabla e_{h}\right) \hat{w}_{h} \mathrm{~d} x-\sum_{F \in \mathcal{F}_{h}^{\circ \partial}} \int_{F} \llbracket \kappa \nabla v_{h} \rrbracket \cdot \boldsymbol{n}_{F} \hat{w}_{h} \mathrm{~d} s-\int_{\partial D} \eta_{h} v_{h} \hat{w}_{h} \mathrm{~d} s,
\end{aligned}
$$

where $\mathcal{F}_{h}^{\circ}$ is the collection of the mesh interfaces that touch the boundary (note that $\hat{w}_{h}$ vanishes on all the remaining interfaces in $\left.\mathcal{F}_{h}^{\circ}\right)$. The Cauchy-Schwarz inequality leads to $\left\|\ell_{h}-\ell \circ T-a_{h}\left(v_{h},(I-T)(\cdot)\right)\right\|_{V_{h}^{\prime}} \leq$ $c \mathfrak{T}_{1} \mathfrak{T}_{2}$ with $\mathfrak{T}_{1}$ and $\mathfrak{T}_{2}$ defined by

$$
\begin{aligned}
& \mathfrak{T}_{1}:=\left(\sum_{K \in \mathcal{T}_{h}^{\partial}} \kappa_{K}^{-1} h_{K}^{2+2 d\left(\frac{1}{2}-\frac{1}{q}\right)}\left\|\nabla \cdot\left(\kappa \nabla e_{h}\right)\right\|_{L^{q}(K)}^{2}+\sum_{F \in \mathcal{F}_{h}^{\circ \partial}} \kappa_{K_{F}}^{-1} \tilde{h}_{F}\left\|\llbracket \kappa \nabla v_{h} \rrbracket \cdot n_{F}\right\|_{L^{2}(F)}^{2}+\left\|\rho_{h}^{\frac{1}{2}} e_{h}\right\|_{L^{2}(\partial D)}^{2}\right)^{\frac{1}{2}}, \\
& \mathfrak{T}_{2}:=\left(\sum_{K \in \mathcal{T}_{h}^{\partial}} \kappa_{K} h_{K}^{-2+2 d\left(\frac{1}{q}-\frac{1}{2}\right)}\left\|\hat{w}_{h}\right\|_{L^{q^{\prime}}(K)}^{2}+\sum_{F \in \mathcal{F}_{h}^{\circ \partial}} \kappa_{K_{F}} \tilde{h}_{F}^{-1}\left\|\hat{w}_{h}\right\|_{L^{2}(F)}^{2}+\left\|\rho_{h}^{\frac{1}{2}} w_{h}\right\|_{L^{2}(\partial D)}^{2}\right)^{\frac{1}{2}},
\end{aligned}
$$

where, for all $F \in \mathcal{F}_{h}^{\circ \partial}, K_{F}$ is the mesh cell sharing $F$ and having the larger value of $\kappa_{K}$ (the choice of $K_{F}$ is irrelevant if both cells give the same value), $\tilde{h}_{F}$ is defined in Lemma 5.7, and where $q^{\prime}$ is the conjugate number of $q$. Moreover, in the last term defining $\mathfrak{T}_{1}$ and $\mathfrak{T}_{2}$, we have exploited the fact that $u$ and $T\left(w_{h}\right)$ have zero trace at the boundary $\partial D$ so that $\left\|\rho_{h}^{\frac{1}{2}} v_{h}\right\|_{L^{2}(\partial D)}=\left\|\rho_{h}^{\frac{1}{2}} e_{h}\right\|_{L^{2}(\partial D)}$ and $\left\|\rho_{h}^{\frac{1}{2}} \hat{w}_{h}\right\|_{L^{2}(\partial D)}=\left\|\eta_{h}^{\frac{1}{2}} w_{h}\right\|_{L^{2}(\partial D)}$. (2a) Bound on $\mathfrak{T}_{1}$. We need to bound $\left\|\llbracket \kappa \nabla v_{h} \rrbracket \cdot \boldsymbol{n}_{F}\right\|_{L^{2}(F)}$ and to this purpose we use Lemma 5.7. This is possible since, by assumption, $\kappa$ is piecewise constant on the mesh $\mathcal{T}_{h}$ and, therefore, $\llbracket \kappa \nabla v_{h} \rrbracket \cdot \boldsymbol{n}_{F} \in P_{F}$. We infer that

$$
\tilde{h}_{F}^{\frac{1}{2}}\left\|\llbracket \kappa \nabla v_{h} \rrbracket \cdot \boldsymbol{n}_{F}\right\|_{L^{2}(F)} \leq c \tilde{h}_{F}^{d\left(\frac{1}{2}-\frac{1}{q}\right)} \sup _{\substack{\varphi \in W_{0}^{1, q^{\prime}}\left(D_{F}\right) \\\|\nabla \varphi\|_{L^{q^{\prime}}\left(D_{F}\right)}=1}} \int_{F} \llbracket \kappa \nabla v_{h} \rrbracket \cdot \boldsymbol{n}_{F} \varphi \mathrm{d} s .
$$


Let $\varphi \in W_{0}^{1, q^{\prime}}\left(D_{F}\right)$ be s.t. $\|\nabla \varphi\|_{\boldsymbol{L}^{q^{\prime}}\left(D_{F}\right)}=1$. By definition of the jump and using the divergence formula, we have

$$
\begin{aligned}
\int_{F} \llbracket \kappa \nabla v_{h} \rrbracket \cdot \boldsymbol{n}_{F} \varphi \mathrm{d} s & =\int_{F}\left(\kappa \nabla v_{h}\right)_{\mid K_{l}} \cdot \boldsymbol{n}_{K_{l}} \varphi \mathrm{d} s+\int_{F}\left(\kappa \nabla v_{h}\right)_{\mid K_{r}} \cdot \boldsymbol{n}_{K_{r}} \varphi \mathrm{d} s \\
& =\sum_{K \in \mathcal{T}_{F}} \int_{K} \nabla \cdot\left(\varphi \kappa \nabla v_{h}\right) \mathrm{d} x=\sum_{K \in \mathcal{T}_{F}} \int_{K}\left(\varphi \nabla \cdot\left(\kappa \nabla v_{h}\right)+\kappa \nabla v_{h} \cdot \nabla \varphi\right) \mathrm{d} x,
\end{aligned}
$$

where $K_{l}, K_{r}$ are the two mesh cells sharing the interface $F$ and where we have set $\mathcal{T}_{F}=\left\{K_{l}, K_{r}\right\}$. Moreover, since $q^{\prime} \geq 2$, the zero-extension of $\varphi$ to $D$ is in $H_{0}^{1}(D)$, and this implies that $\sum_{K \in \mathcal{T}_{F}} \int_{K}(\varphi \nabla \cdot(\kappa \nabla u)+$ $\kappa \nabla u \cdot \nabla \varphi) \mathrm{d} x=0$. Combining these two relations, we infer that

$$
\int_{F} \llbracket \kappa \nabla v_{h} \rrbracket \cdot \boldsymbol{n}_{F} \varphi \mathrm{d} s=\sum_{K \in \mathcal{T}_{F}} \int_{K}\left(\varphi \nabla \cdot\left(\kappa \nabla e_{h}\right)+\kappa \nabla e_{h} \cdot \nabla \varphi\right) \mathrm{d} x .
$$

Invoking Hölder's inequality and the Poincaré-Steklov inequality in $W_{0}^{1, q^{\prime}}\left(D_{F}\right)$, which implies that $\|\varphi\|_{L^{q^{\prime}}(K)} \leq$ $c h_{K}\|\nabla \varphi\|_{\boldsymbol{L}^{q^{\prime}\left(D_{F}\right)}}=c h_{K}$ for all $K \in \mathcal{T}_{F}$, we infer that

$$
\left|\int_{K} \varphi \nabla \cdot\left(\kappa \nabla e_{h}\right) \mathrm{d} x\right| \leq\|\varphi\|_{L^{q^{\prime}}(K)}\left\|\nabla \cdot\left(\kappa \nabla e_{h}\right)\right\|_{L^{q}(K)} \leq c h_{K}\left\|\nabla \cdot\left(\kappa \nabla e_{h}\right)\right\|_{L^{q}(K)} .
$$

Moreover, invoking Hölder's inequality together with $\|\nabla \varphi\|_{\boldsymbol{L}^{q^{\prime}(K)}} \leq\|\nabla \varphi\|_{\boldsymbol{L}^{q^{\prime}\left(D_{F}\right)}}=1$ for all $K \in \mathcal{T}_{F}, q \leq 2$, and the shape-regularity of the mesh sequence, we infer that

$$
\left|\int_{K} \kappa \nabla e_{h} \cdot \nabla \varphi \mathrm{d} x\right| \leq\|\nabla \varphi\|_{\boldsymbol{L}^{q^{\prime}(K)}}\left\|\kappa \nabla e_{h}\right\|_{\boldsymbol{L}^{q}(K)} \leq c h_{K}^{d\left(\frac{1}{q}-\frac{1}{2}\right)}\left\|\kappa \nabla e_{h}\right\|_{\boldsymbol{L}^{2}(K)} .
$$

Putting the above bounds together and since $K_{F}$ has been chosen so that $\kappa_{K_{F}}=\max _{K \in \mathcal{T}_{F}} \kappa_{K}$, we conclude that $\mathfrak{T}_{1} \leq c\left\|e_{h}\right\|_{V_{\sharp}}$.

(2b) Bound on $\mathfrak{T}_{2}$. Applying a inverse inequality from $L^{q^{\prime}}(K)$ to $L^{2}(K)$, for all $K \in \mathcal{T}_{h}^{\partial}$, we infer that $h_{K}^{-1+d\left(\frac{1}{q}-\frac{1}{2}\right)}\left\|\hat{w}_{h}\right\|_{L^{q^{\prime}}(K)}=h_{K}^{-1+d\left(\frac{1}{2}-\frac{1}{q^{\prime}}\right)}\left\|\hat{w}_{h}\right\|_{L^{q^{\prime}}(K)} \leq c h_{K}^{-1}\left\|\hat{w}_{h}\right\|_{L^{2}(K)}$. Moreover, applying an inverse trace inequality on $K_{F}$, for all $F \in \mathcal{F}_{h}^{\circ \partial}$, and invoking the shape-regularity of the mesh sequence, we infer that $\tilde{h}_{F}^{-\frac{1}{2}}\left\|\hat{w}_{h}\right\|_{L^{2}(F)} \leq c h_{K_{F}}^{-1}\left\|\hat{w}_{h}\right\|_{L^{2}\left(K_{F}\right)}$. Finally, using the approximation property (6.2) on all $K \in \mathcal{T}_{h}^{\partial}$ and recalling the definition of the contrast factor $\xi_{\kappa}$, we conclude that $\mathfrak{T}_{2} \leq c \xi_{\kappa}^{\frac{1}{2}}\left\|w_{h}\right\|_{V_{h}}$. This completes the proof of (4.17).

\subsection{Mollified error estimate}

We are going to assume in this section that there is a real number $r>0$ so that the exact solution $u$ is in $H^{1+r}(D)$. Let $k \geq 1$ be the degree of the underlying finite elements. Let us set $t:=\min (r, k)$. If $2 t \geq d$, let $p$ be any real number larger than 2 . If $2 t<d$ let us set $p=\frac{2 d}{d-2 t}$; clearly $p>2$ since $t>0$. Let us now consider some real number $q$ such that $q>\frac{2 d}{2+d}$. We define the functional space

$$
V_{\mathrm{S}}:=\left\{v \in H^{1}(D) \mid \boldsymbol{\sigma}(v) \in \boldsymbol{L}^{p}(D), \nabla \cdot \boldsymbol{\sigma}(v) \in L^{q}(D)\right\},
$$

with the shorthand notation $\boldsymbol{\sigma}(v):=-\kappa \nabla v$ for all $v \in H^{1}(D)$. Notice that the pair $(p, q)$ satisfies the requirements in (5.8).

Lemma 6.2 (Exact solution). If $u \in H^{1+r}(D), r>0$, and if the source term $f$ is in $L^{q}(D)$ with $q>\frac{2 d}{2+d}$, then $u$ is in $V_{\mathrm{S}}$ as defined by (6.6).

Proof. Owing to the Sobolev Embedding Theorem (see e.g., $[9, \S 9.3]$ ), we infer that $\boldsymbol{H}^{t}(D) \hookrightarrow \boldsymbol{L}^{p}(D)$ (indeed, if $2 t<d$, we have $\boldsymbol{H}^{t}(D) \hookrightarrow \boldsymbol{L}^{s}(D)$ for all $s \in\left[2, \frac{2 d}{d-2 t}\right]=[2, p]$, whereas if $2 t \geq d$, we have $\boldsymbol{H}^{t}(D) \hookrightarrow \boldsymbol{H}^{\frac{d}{2}}(D) \hookrightarrow \boldsymbol{L}^{s}(D)$ for all $s \in[2, \infty)$, and choosing $s=p$ again yields $\left.\boldsymbol{H}^{t}(D) \hookrightarrow \boldsymbol{L}^{p}(D)\right)$. Since $r \geq t$, we infer that $\boldsymbol{H}^{r}(D) \hookrightarrow \boldsymbol{H}^{t}(D)$, so that the above argument implies that $\nabla u \in \boldsymbol{L}^{p}(D)$, and since $\kappa$ is piecewise constant and $\boldsymbol{\sigma}(u)=-\kappa \nabla u$, we have $\boldsymbol{\sigma}(u) \in \boldsymbol{L}^{p}(D)$. Moreover, since $\nabla \cdot \boldsymbol{\sigma}(u)=f$ and $f \in L^{q}(D)$ with $q>\frac{2 d}{2+d}$ by assumption, we have $\nabla \cdot \boldsymbol{\sigma}(u) \in L^{q}(D)$. In conclusion, $u \in V_{\mathrm{S}}$. 
We are now ready to perform the error analysis. We consider the setting of $\S 4.3$ and we want to apply Lemma 4.8. We set $V_{\sharp}:=V_{\mathrm{S}}+V_{h}$ that we equip with the norm

$$
\|v\|_{V_{\sharp}}^{2}:=\|v\|_{V_{b}}^{2}+\sum_{K \in \overline{\mathcal{T}}_{h}^{\partial}} \kappa_{K}^{-1}\left(h_{K}^{d\left(\frac{1}{2}-\frac{1}{p}\right)}\|\kappa \nabla v\|_{L^{p}(K)}+h_{K}^{1+d\left(\frac{1}{2}-\frac{1}{q}\right)}\|\nabla \cdot(\kappa \nabla v)\|_{L^{q}(K)}\right)^{2},
$$

where $\overline{\mathcal{T}}_{h}^{\partial}$ is the collection of all the mesh cells having a boundary face, i.e., $\overline{\mathcal{T}}_{h}^{\partial}:=\cup_{F \in \mathcal{F}_{h}^{\partial}}\left\{K_{F}\right\}$. Compared with the norm defined by (6.5) used for the trimmed error estimate, we observe that there is now an additional term measuring $\kappa \nabla v$ in the $\boldsymbol{L}^{p}$-norm, but the summation is now restricted to the smaller set $\overline{\mathcal{T}}_{h}^{\partial} \subsetneq \mathcal{T}_{h}^{\partial}$. Notice also that (4.11) holds true with $c_{b}=1$. We define the following bilinear form on $V_{\sharp} \times V_{h}$ :

$$
a_{\sharp}\left(v, w_{h}\right):=\int_{D} \kappa \nabla v \cdot \nabla w \mathrm{~d} x-\sum_{F \in \mathcal{F}_{h}^{\partial}}\left\langle\left(\boldsymbol{\sigma}(v)_{\mid K_{F}} \cdot \boldsymbol{n}\right)_{\mid F}, w_{h}\right\rangle,
$$

recalling that for all $F \in \mathcal{F}_{h}^{\partial}, \boldsymbol{n}_{K_{F}}=\boldsymbol{n}$ (the unit outward normal to $D$ ), and the action of the linear form $\left\langle\left(\boldsymbol{\sigma}(v)_{\mid K_{F}} \cdot \boldsymbol{n}\right)_{\mid F}, \cdot\right\rangle$ has been defined in (5.11) for all $F \in \mathcal{F}_{h}^{\partial}$.

Lemma 6.3 (Mollified error estimate). The assumptions of Lemma 4.8 hold true for the bilinear form $a_{\sharp}$ defined by (6.8) and the stabilization bilinear form $s_{h}$ defined by (3.6b). Moreover, the constant $\omega_{V_{\sharp}, W_{h}}^{\text {mol }}$ involved in (4.20) and the constant $\sigma_{V_{h}, W_{h}}$ involved in (4.22) are independent of the contrast in $\kappa$.

Proof. (1) Proof of (4.20). This is a direct consequence of (5.13), the Cauchy-Schwarz inequality, the choice (3.7) of the penalty parameter $\rho_{h}$, and the fact that $\left\|\rho_{h}^{\frac{1}{2}} w_{h}\right\|_{L^{2}(\partial D)} \leq\left\|w_{h}\right\|_{V_{h}}$ for all $w_{h} \in V_{h}$.

(2) Proof of (4.21a). Let $v_{h}, w_{h} \in V_{h}$. Let $F \in \mathcal{F}_{h}^{\partial}$ and let $K_{F}$ be the mesh cell having $F$ as a boundary face. Since the restriction of $\boldsymbol{\sigma}\left(v_{h}\right)$ to $K_{F}$ is smooth and since the restriction of $E_{F}^{K_{F}}\left(w_{h}\right)$ is nonzero only on the face $F$ of $K_{F}$, we have

$$
\begin{aligned}
\left\langle\left(\boldsymbol{\sigma}\left(v_{h}\right)_{\mid K_{F}} \cdot \boldsymbol{n}\right)_{\mid F}, w_{h}\right\rangle & =\int_{K_{F}}\left(\boldsymbol{\sigma}\left(v_{h}\right) \cdot E_{F}^{K_{F}}\left(w_{h}\right)+\left(\nabla \cdot \boldsymbol{\sigma}\left(v_{h}\right)\right) E_{F}^{K_{F}}\left(w_{h}\right)\right) \mathrm{d} x \\
& =\int_{\partial K_{F}}\left(\boldsymbol{\sigma}\left(v_{h}\right)_{\mid K_{F}} \cdot \boldsymbol{n}_{K_{F}}\right) E_{F}^{K_{F}}\left(w_{h}\right) \mathrm{d} s \\
& =\int_{F}\left(\boldsymbol{\sigma}\left(v_{h}\right)_{\mid K_{F}} \cdot \boldsymbol{n}\right) w_{h} \mathrm{~d} s
\end{aligned}
$$

where we have used the divergence formula in $K_{F}$ and where we have dropped the restriction to $K_{F}$ in the integral over $K_{F}$ to alleviate the notation. Summing over all the boundary faces and recalling the definition (3.6) of $\tilde{a}_{h}$, we conclude that (4.21a) holds true.

(3) Proof of (4.21b). Let $w_{h} \in V_{h}$ and let $v \in V_{\mathrm{s}}$. Let $\mathcal{K}_{\delta}^{\mathrm{d}}: \boldsymbol{L}^{1}(D) \rightarrow \boldsymbol{C}^{\infty}(\bar{D})$ and $\mathcal{K}_{\delta}^{\mathrm{b}}: L^{1}(D) \rightarrow C^{\infty}(\bar{D})$ be the mollification operators introduced in $\S 5.1$. Recall the following key commuting property:

$$
\nabla \cdot\left(\mathcal{K}_{\delta}^{\mathrm{d}}(\boldsymbol{\tau})\right)=\mathcal{K}_{\delta}^{\mathrm{b}}(\nabla \cdot \boldsymbol{\tau})
$$

for all $\boldsymbol{\tau} \in \boldsymbol{L}^{1}(D)$ s.t. $\nabla \cdot \boldsymbol{\tau} \in L^{1}(D)$. It is important to realize that this property can be applied to $\boldsymbol{\sigma}(v)$ since $\nabla \cdot \boldsymbol{\sigma}(v) \in L^{1}(D)$ by definition of $V_{\mathrm{s}}$. Let us consider the mollified bilinear form

$$
n_{\sharp \delta}\left(v, w_{h}\right):=\sum_{F \in \mathcal{F}_{h}^{\partial}}\left\langle\left(\mathcal{K}_{\delta}^{\mathrm{d}}(\boldsymbol{\sigma}(v))_{\mid K_{F}} \cdot \boldsymbol{n}\right)_{\mid F}, w_{h}\right\rangle .
$$

Owing to the commuting property (6.9), we infer that

$$
\left\langle\left(\mathcal{K}_{\delta}^{\mathrm{d}}(\boldsymbol{\sigma}(v))_{\mid K_{F}} \cdot \boldsymbol{n}\right)_{\mid F}, w_{h}\right\rangle=\int_{K_{F}}\left(\mathcal{K}_{\delta}^{\mathrm{d}}(\boldsymbol{\sigma}(v)) \cdot E_{F}^{K_{F}}\left(w_{h}\right)+\mathcal{K}_{\delta}^{\mathrm{b}}(\nabla \cdot \boldsymbol{\sigma}(v)) E_{F}^{K_{F}}\left(w_{h}\right)\right) \mathrm{d} x .
$$

Letting $\delta \downarrow 0$, Theorem 5.3 implies that

$$
\begin{aligned}
\int_{K_{F}}\left(\mathcal{K}_{\delta}^{\mathrm{d}}(\boldsymbol{\sigma}(v)) \cdot E_{F}^{K_{F}}\left(w_{h}\right)+\mathcal{K}_{\delta}^{\mathrm{b}}((\nabla \cdot \boldsymbol{\sigma}(v))) E_{F}^{K_{F}}\left(w_{h}\right)\right) \mathrm{d} x \rightarrow \\
\int_{K_{F}}\left(\boldsymbol{\sigma}(v) \cdot E_{F}^{K_{F}}\left(w_{h}\right)+(\nabla \cdot \boldsymbol{\sigma}(v)) E_{F}^{K_{F}}\left(w_{h}\right)\right) \mathrm{d} x=\left\langle\left(\boldsymbol{\sigma}(v)_{\mid K_{F}} \cdot \boldsymbol{n}\right)_{\mid F}, w_{h}\right\rangle .
\end{aligned}
$$


Summing over the mesh boundary faces, we infer that

$$
n_{\sharp \delta}\left(v, w_{h}\right) \rightarrow \int_{D} \kappa \nabla v \cdot \nabla w_{h} \mathrm{~d} x-a_{\sharp}\left(v, w_{h}\right) \quad \text { as } \delta \downarrow 0 .
$$

Moreover, since the mollified function $\mathcal{K}_{\delta}^{\mathrm{d}}(\boldsymbol{\sigma}(v))$ is smooth, by repeating the calculation done in Step (2), we also have

$$
n_{\sharp \delta}\left(v, w_{h}\right)=\sum_{F \in \mathcal{F}_{h}^{\partial}} \int_{F}\left(\mathcal{K}_{\delta}^{\mathrm{d}}(\boldsymbol{\sigma}(v)) \cdot \boldsymbol{n}\right) w_{h} \mathrm{~d} s .
$$

Since $\llbracket \mathcal{K}_{\delta}^{\mathrm{d}}(\boldsymbol{\sigma}(v)) \rrbracket \cdot \boldsymbol{n}_{F}=\llbracket w_{h} \rrbracket=0$ for all $F \in \mathcal{F}_{h}^{\circ}$, we obtain

$$
n_{\sharp \delta}\left(v, w_{h}\right)=\sum_{K \in \mathcal{T}_{h}} \int_{\partial K} \mathcal{K}_{\delta}^{\mathrm{d}}(\boldsymbol{\sigma}(v))_{\mid K} \cdot \boldsymbol{n}_{K} w_{h} \mathrm{~d} s=\int_{D}\left(\mathcal{K}_{\delta}^{\mathrm{d}}(\boldsymbol{\sigma}(v)) \cdot \nabla w_{h}+\mathcal{K}_{\delta}^{\mathrm{b}}(\nabla \cdot \boldsymbol{\sigma}(v)) w_{h}\right) \mathrm{d} x,
$$

where we used the divergence formula in each mesh cell $K$ and the commuting property (6.9). Letting $\delta \downarrow 0$ and invoking again Theorem 5.3 shows that

$$
n_{\sharp \delta}\left(v, w_{h}\right) \rightarrow \int_{D}\left(\boldsymbol{\sigma}(v) \cdot \nabla w_{h}+(\nabla \cdot \boldsymbol{\sigma}(v)) w_{h}\right) \mathrm{d} x=-\int_{D}\left(\kappa \nabla v \cdot \nabla w_{h}+(\nabla \cdot(\kappa \nabla v)) w_{h}\right) \mathrm{d} x .
$$

The proof of (4.21b) follows by identifying the two limits of $n_{\sharp \delta}\left(v, w_{h}\right)$ and since $\int_{D}(\nabla \cdot(\kappa \nabla u)) w_{h} \mathrm{~d} x=$ $\ell_{h}\left(w_{h}\right)$.

(4) Proof of (4.22). The Cauchy-Schwarz inequality implies that $\left\|s_{h}\left(v_{h}, \cdot\right)\right\|_{V_{h}^{\prime}} \leq \eta_{0}\left\|\rho_{h}^{\frac{1}{2}} v_{h}\right\|_{L^{2}(\partial D)}$ for all $v_{h} \in V_{h}$, and (4.22) follows since any function $v$ in $V=H_{0}^{1}(D)$ has a zero trace on $\partial D$.

\section{Application to the time-harmonic Maxwell's equations}

In this section, we show how the trimmed error estimate from Lemma 4.6 and the mollified error estimate from Lemma 4.8 can be applied to the approximation of the time-harmonic Maxwell's equations using the boundary penalty method described in Section 3.3. The discrete space is $\boldsymbol{V}_{h}=\boldsymbol{P}^{\mathrm{g}}\left(\mathcal{T}_{h}\right)$, and the space $\boldsymbol{V}_{\mathrm{b}}:=\boldsymbol{H}_{0}(\operatorname{curl} ; D)+\boldsymbol{V}_{h}$ can be equipped with the norm $\|\cdot\|_{\boldsymbol{V}_{\mathrm{b}}}$ that extends to $\boldsymbol{V}_{\mathrm{b}}$ the norm $\|\cdot\|_{\boldsymbol{V}_{h}}$ originally defined by (3.13) on $\boldsymbol{V}_{h}$; notice in particular that functions in $\boldsymbol{V}_{b}$ have a well-defined tangential trace on $\partial D$. Indeed, any function $\boldsymbol{b} \in \boldsymbol{V}_{\mathrm{b}}$ can be written as $\boldsymbol{b}=\boldsymbol{b}_{0}+\boldsymbol{b}_{h}$ with $\boldsymbol{b}_{0} \in \boldsymbol{H}_{0}(\operatorname{curl} ; D)$ and $\boldsymbol{b}_{h} \in \boldsymbol{V}_{h}$, and we have $\gamma^{\mathrm{c}}(\boldsymbol{b})=\boldsymbol{b}_{h \mid \partial D} \times \boldsymbol{n}$ so that $\left\|\rho_{h}^{\frac{1}{2}} \boldsymbol{b}\right\|_{\boldsymbol{L}^{2}(\partial D)}=\left\|\rho_{h}^{\frac{1}{2}} \boldsymbol{b}_{h}\right\|_{\boldsymbol{L}^{2}(\partial D)}$. Finally, the discrete forms $a_{h}(\cdot, \cdot)$ and $\ell_{h}(\cdot)$ are defined by $(3.11)$. The constants in the error estimates derived in this section depend on the shape-regularity of the mesh sequence and on the reference finite element. These constants can also depend on the local ratios $\mu_{\sharp, K} / \mu_{r, K}$ and $\kappa_{\sharp, K} / \kappa_{r, K}$ for all $K \in \mathcal{T}_{h}$; for simplicity, we will not track these dependencies in what follows. Notice that these ratios are equal to 1 when the coefficients $\widetilde{\mu}$ and $\kappa$ are real.

\subsection{Trimmed error estimate}

We define the trimming operator $T: \boldsymbol{P}^{\mathrm{g}}\left(\mathcal{T}_{h}\right) \rightarrow \boldsymbol{P}^{\mathrm{g}}\left(\mathcal{T}_{h}\right) \cap \boldsymbol{H}_{0}(\operatorname{curl} ; D)=\left\{\boldsymbol{b}_{h} \in \boldsymbol{P}^{\mathrm{g}}\left(\mathcal{T}_{h}\right) \mid \boldsymbol{b}_{h \mid \partial D} \times \boldsymbol{n}=\mathbf{0}\right\}$ such that, for all $\boldsymbol{b}_{h} \in \boldsymbol{P}^{\mathrm{g}}\left(\mathcal{T}_{h}\right), T\left(\boldsymbol{b}_{h}\right)_{\mid K}$, for all $K \in \mathcal{T}_{h}$, is defined by zeroing out all the degrees of freedom of the tangential component of $\boldsymbol{b}_{h}$ at the boundary. Note that the trimming operator couples the Cartesian components of $\boldsymbol{b}_{h}$ if the faces composing the boundary $\partial D$ are not orthogonal to the coordinate axes. We have $T\left(\boldsymbol{b}_{h}\right)=\boldsymbol{b}_{h}$ on all $K \in \mathcal{T}_{h} \backslash \mathcal{T}_{h}^{\partial}$, whereas for all $K \in \mathcal{T}_{h}^{\partial}$, one can prove the following bounds for all $\boldsymbol{b}_{h} \in \boldsymbol{P}^{\mathrm{g}}\left(\mathcal{T}_{h}\right)$ with $c$ uniform w.r.t. $h$ : If $\partial K \cap \partial D$ is composed of one or more boundary faces, then

$$
h_{K}\left\|\nabla\left(\boldsymbol{b}_{h}-T\left(\boldsymbol{b}_{h}\right)\right)\right\|_{\boldsymbol{L}^{2}(K)}+\left\|\boldsymbol{b}_{h}-T\left(\boldsymbol{b}_{h}\right)\right\|_{\boldsymbol{L}^{2}(K)} \leq c h_{K}^{\frac{1}{2}}\left\|\boldsymbol{b}_{h} \times \boldsymbol{n}\right\|_{\boldsymbol{L}^{2}(\partial K \cap \partial D)},
$$

whereas if $\partial K \cap \partial D$ is a manifold of dimension $d^{\prime}<d-1$, then

$$
h_{K}\left\|\nabla\left(\boldsymbol{b}_{h}-T\left(\boldsymbol{b}_{h}\right)\right)\right\|_{\boldsymbol{L}^{2}(K)}+\left\|\boldsymbol{b}_{h}-T\left(\boldsymbol{b}_{h}\right)\right\|_{L^{2}(K)} \leq c h_{K}^{\frac{1}{2}}\left\|\boldsymbol{b}_{h} \times \boldsymbol{n}\right\|_{\boldsymbol{L}^{2}(F)}, \quad \forall F \in \mathcal{F}_{K}^{\partial},
$$


where we recall that $\mathcal{F}_{K}^{\partial}$ is the collection of the boundary faces containing the manifold $\partial K \cap \partial D$. We introduce the contrast factor $\xi_{\kappa_{r}}$ for the parameter $\kappa_{r}$ which is defined similarly to (6.3) by replacing $\kappa$ by $\kappa_{r}$. We also define the local magnetic Reynolds numbers $\zeta_{\mu \kappa, F}:=\mu_{r, K_{F}} h_{K_{F}}^{2} / \kappa_{r, K_{F}}$, for all $F \in \mathcal{F}_{h}^{\partial}$. Finally, let us set

$$
\boldsymbol{V}_{\mathrm{S}}:=\left\{v \in \boldsymbol{H}_{0}(\operatorname{curl} ; D) \mid \nabla \times(\kappa \nabla \times v) \in \boldsymbol{L}^{2}(D)\right\},
$$

and let us equip the space $\boldsymbol{V}_{\sharp}:=\boldsymbol{V}_{\mathrm{S}}+\boldsymbol{V}_{h}$ with the norm

$$
\|\boldsymbol{b}\|_{\boldsymbol{V}_{\sharp}}:=\left(\|\boldsymbol{b}\|_{\boldsymbol{V}_{\boldsymbol{b}}}^{2}+\sum_{K \in \mathcal{T}_{h}^{\partial}} \kappa_{r, K}^{-1} h_{K}^{2}\|\nabla \times(\kappa \nabla \times \boldsymbol{b})\|_{\boldsymbol{L}^{2}(K)}^{2}\right)^{\frac{1}{2}} .
$$

Lemma 7.1 (Trimmed error estimate). The assumptions of Lemma 4.6 hold true with the trimming operator $T: \boldsymbol{P}^{\mathrm{g}}\left(\mathcal{T}_{h}\right) \rightarrow \boldsymbol{P}^{\mathrm{g}}\left(\mathcal{T}_{h}\right) \cap \boldsymbol{H}_{0}(\mathrm{curl} ; D)$ defined above, where the constants $\omega_{\boldsymbol{V}_{\sharp}, \boldsymbol{V}_{h}}^{\mathrm{tri}}$ and $\varpi_{\boldsymbol{V}_{\sharp}, \boldsymbol{V}_{h}}^{\mathrm{tri}}$ are proportional to $\xi_{\kappa_{r}}^{\frac{1}{2}}$ and to $\max \left(1, \zeta_{\mu \kappa}^{\frac{1}{2}}\right)$, where $\xi_{\kappa_{r}}$ is the contrast factor for $\kappa_{r}$ and $\zeta_{\mu \kappa}:=\max _{F \in \mathcal{F}_{h}^{\partial}} \zeta_{\mu \kappa, F}$ where $\zeta_{\mu \kappa, F}$ is the local magnetic Reynolds number associated with the boundary face $F$.

Proof. We only highlight the differences with respect to the proof of Lemma 6.1.

(1) Verification of (4.16). Let $\left(\boldsymbol{v}_{h}, \boldsymbol{b}_{h}\right) \in \boldsymbol{V}_{h} \times \boldsymbol{V}_{h}$. Since $T\left(\boldsymbol{b}_{h}\right) \in \boldsymbol{H}_{0}(\operatorname{curl} ; D)$, we infer that

$$
\begin{aligned}
a\left(\boldsymbol{A}, T\left(\boldsymbol{b}_{h}\right)\right)-a_{h}\left(\boldsymbol{v}_{h}, T\left(\boldsymbol{b}_{h}\right)\right) & =\int_{D}\left(\widetilde{\mu}\left(\boldsymbol{A}-\boldsymbol{v}_{h}\right) \cdot \overline{T\left(\boldsymbol{b}_{h}\right)}+\kappa \nabla \times\left(\boldsymbol{A}-\boldsymbol{v}_{h}\right) \cdot \nabla \times \overline{T\left(\boldsymbol{b}_{h}\right)}\right) \mathrm{d} x \\
& \leq\left\|\boldsymbol{A}-\boldsymbol{v}_{h}\right\|_{\boldsymbol{V}_{b}}\left(\left\|\mu_{r}^{\frac{1}{2}} T\left(\boldsymbol{b}_{h}\right)\right\|_{\boldsymbol{L}^{2}(D)}+\left\|\kappa_{r}^{\frac{1}{2}} \nabla \times T\left(\boldsymbol{b}_{h}\right)\right\|_{\boldsymbol{L}^{2}(D)}\right) .
\end{aligned}
$$

Since $\left\|\boldsymbol{A}-\boldsymbol{v}_{h}\right\|_{\boldsymbol{V}_{b}} \leq\left\|\boldsymbol{A}-\boldsymbol{v}_{h}\right\|_{\boldsymbol{V}_{\sharp}}$, we just have to prove that $\left\|\mu_{r}^{\frac{1}{2}} T\left(\boldsymbol{b}_{h}\right)\right\|_{\boldsymbol{L}^{2}(D)}+\left\|\kappa_{r}^{\frac{1}{2}} \nabla \times T\left(\boldsymbol{b}_{h}\right)\right\|_{\boldsymbol{L}^{2}(D)} \leq$ $c\left\|\boldsymbol{w}_{h}\right\|_{\boldsymbol{V}_{h}}$. We have $T\left(\boldsymbol{b}_{h}\right)=\boldsymbol{b}_{h}$ on all $K \in \mathcal{T}_{h} \backslash \mathcal{T}_{h}^{\partial}$, so that we only need to bound $T\left(\boldsymbol{b}_{h}\right)$ on all $K \in \mathcal{T}_{h}^{\partial}$. Reasoning as in the proof of Lemma 6.1 and estimating the approximation properties of $\nabla \times T\left(\boldsymbol{b}_{h}\right)$ by those of $\nabla T\left(\boldsymbol{b}_{h}\right)$, we infer that, for all $K \in \mathcal{T}_{h}^{\partial}$, if $\partial K \cap \partial D$ is composed of one or more boundary faces, then

$$
\left\|\kappa_{r}^{\frac{1}{2}} \nabla \times T\left(\boldsymbol{b}_{h}\right)\right\|_{\boldsymbol{L}^{2}(K)} \leq\left\|\kappa_{r}^{\frac{1}{2}} \nabla \times \boldsymbol{b}_{h}\right\|_{\boldsymbol{L}^{2}(K)}+c\left\|\rho_{h}^{\frac{1}{2}}\left(\boldsymbol{b}_{h} \times \boldsymbol{n}\right)\right\|_{\boldsymbol{L}^{2}(\partial K \cap \partial D)},
$$

whereas if the manifold $\partial K \cap \partial D$ is of dimension $d^{\prime}<d-1$, then

$$
\left\|\kappa_{r}^{\frac{1}{2}} \nabla \times T\left(\boldsymbol{b}_{h}\right)\right\|_{L^{2}(K)} \leq\left\|\kappa_{r}^{\frac{1}{2}} \nabla \times \boldsymbol{b}_{h}\right\|_{\boldsymbol{L}^{2}(K)}+c \xi_{\kappa_{r}}^{\frac{1}{2}}\left\|\rho_{h}^{\frac{1}{2}}\left(\boldsymbol{b}_{h} \times \boldsymbol{n}\right)\right\|_{\boldsymbol{L}^{2}(F)},
$$

where $F$ is a boundary face in $\mathcal{F}_{K}^{\partial}$ s.t. $\kappa_{r, K_{F}}$ is maximal. The reasoning to bound $\left\|\mu_{r}^{\frac{1}{2}} T\left(\boldsymbol{b}_{h}\right)\right\|_{L^{2}(K)}$ for all $K \in \mathcal{T}_{h}^{\partial}$ is similar and leads to the additional dependency on the factor $\max \left(1, \zeta_{\mu \kappa}\right)$.

(2) Verification of (4.17). Let $\left(\boldsymbol{v}_{h}, \boldsymbol{b}_{h}\right) \in \boldsymbol{V}_{h} \times \boldsymbol{V}_{h}$ and let us set $\boldsymbol{e}_{h}:=\boldsymbol{A}-\boldsymbol{v}_{h}$ and $\boldsymbol{d}_{h}:=\boldsymbol{b}_{h}-T\left(\boldsymbol{b}_{h}\right)$. A direct calculation shows that

$$
\begin{aligned}
& \ell_{h}\left(\boldsymbol{b}_{h}\right)-\ell\left(T\left(\boldsymbol{b}_{h}\right)\right)-a_{h}\left(\boldsymbol{v}_{h},(I-T)\left(\boldsymbol{b}_{h}\right)\right) \\
& =\int_{D} \boldsymbol{f} \cdot \overline{\boldsymbol{d}}_{h} \mathrm{~d} x-\int_{D}\left(\widetilde{\mu} \boldsymbol{v}_{h} \cdot \overline{\boldsymbol{d}}_{h}+\kappa \nabla \times \boldsymbol{v}_{h} \cdot \nabla \times \overline{\boldsymbol{d}}_{h}\right) \mathrm{d} x-\int_{\partial D}\left(\boldsymbol{n} \times\left(\kappa \nabla \times \boldsymbol{v}_{h}\right)\right) \cdot \overline{\boldsymbol{d}}_{h} \mathrm{~d} s-\int_{\partial D} \eta_{h}\left(\boldsymbol{v}_{h} \times \boldsymbol{n}\right) \cdot\left(\overline{\boldsymbol{d}}_{h} \times \boldsymbol{n}\right) \mathrm{d} s \\
& =\sum_{K \in \mathcal{T}_{h}^{\partial}} \int_{K}\left(\widetilde{\mu} \boldsymbol{e}_{h} \cdot \overline{\boldsymbol{d}}_{h}+\nabla \times\left(\kappa \nabla \times \boldsymbol{e}_{h}\right) \cdot \overline{\boldsymbol{d}}_{h}\right) \mathrm{d} x+\sum_{F \in \mathcal{F}_{h}^{\circ \partial}} \int_{F} \boldsymbol{n}_{F} \times \llbracket \kappa \nabla \times \boldsymbol{v}_{h} \rrbracket \cdot \overline{\boldsymbol{d}}_{h} \mathrm{~d} s-\int_{\partial D} \eta_{h}\left(\boldsymbol{v}_{h} \times \boldsymbol{n}\right) \cdot\left(\overline{\boldsymbol{d}}_{h} \times \boldsymbol{n}\right) \mathrm{d} s,
\end{aligned}
$$

where we recall that $\mathcal{F}_{h}^{\circ \partial}$ is the collection of the mesh interfaces that touch the boundary. The CauchySchwarz inequality leads to $\left\|\ell_{h}-\ell \circ T-a_{h}\left(\boldsymbol{v}_{h},(I-T)(\cdot)\right)\right\|_{\boldsymbol{V}_{h}^{\prime}} \leq c \mathfrak{T}_{1} \mathfrak{T}_{2}$ with $\mathfrak{T}_{1}$ and $\mathfrak{T}_{2}$ defined by

$$
\begin{aligned}
\mathfrak{T}_{1}= & \left(\sum_{K \in \mathcal{T}_{h}^{\partial}} \mu_{r, K}\left\|\boldsymbol{e}_{h}\right\|_{\boldsymbol{L}^{2}(K)}^{2}+\kappa_{r, K}^{-1} h_{K}^{2}\left\|\nabla \times\left(\kappa \nabla \times \boldsymbol{e}_{h}\right)\right\|_{\boldsymbol{L}^{2}(K)}^{2}+\sum_{F \in \mathcal{F}_{h}^{\circ}} \kappa_{r, K_{F}}^{-1} \tilde{h}_{F}\left\|\llbracket \kappa \nabla \times \boldsymbol{v}_{h} \rrbracket \times \boldsymbol{n}_{F}\right\|_{\boldsymbol{L}^{2}(F)}^{2}\right. \\
& \left.+\left\|\rho_{h}^{\frac{1}{2}}\left(\boldsymbol{v}_{h} \times \boldsymbol{n}\right)\right\|_{\boldsymbol{L}^{2}(\partial D)}^{2}\right)^{\frac{1}{2}} \\
\mathfrak{T}_{2}= & \left(\sum_{K \in \mathcal{T}_{h}^{\partial}}\left(\mu_{r, K}+\kappa_{r, K} h_{K}^{-2}\right)\left\|\boldsymbol{d}_{h}\right\|_{\boldsymbol{L}^{2}(K)}^{2}+\sum_{F \in \mathcal{F}_{h}^{\circ o}} \kappa_{r, K_{F}} \tilde{h}_{F}^{-1}\left\|\boldsymbol{d}_{h}\right\|_{\boldsymbol{L}^{2}(F)}^{2}+\left\|\rho_{h}^{\frac{1}{2}}\left(\boldsymbol{b}_{h} \times \boldsymbol{n}\right)\right\|_{\boldsymbol{L}^{2}(\partial D)}^{2}\right)^{\frac{1}{2}},
\end{aligned}
$$


where, for all $F \in \mathcal{F}_{h}^{\circ}, K_{F}$ is the mesh cell sharing $F$ and having the larger value of $\kappa_{r, K}$ (the choice of $K_{F}$ is irrelevant if both cells give the same value), and $\tilde{h}_{F}$ is defined in Lemma 5.7.

(2a) Bound on $\mathfrak{T}_{1}$. The bound on the terms composing the summation over $K \in \mathcal{T}_{h}^{\partial}$ is straightforward. To bound $\left\|\llbracket \kappa \nabla \times \boldsymbol{v}_{h} \rrbracket \times \boldsymbol{n}_{F}\right\|_{\boldsymbol{L}^{2}(F)}$, for all $F \in \mathcal{F}_{h}^{\circ \partial}$, we use Lemma 5.7 (with $p=2$ ). This is possible since, by assumption, $\kappa$ is piecewise constant on the mesh $\mathcal{T}_{h}$ and, therefore, $\llbracket \kappa \nabla \times \boldsymbol{v}_{h} \rrbracket \times \boldsymbol{n}_{F} \in \boldsymbol{P}_{F}$. Finally, $\left\|\rho_{h}^{\frac{1}{2}}\left(\boldsymbol{v}_{h} \times \boldsymbol{n}\right)\right\|_{\boldsymbol{L}^{2}(F)}=\left\|\rho_{h}^{\frac{1}{2}}\left(\boldsymbol{e}_{h} \times \boldsymbol{n}\right)\right\|_{\boldsymbol{L}^{2}(F)}$, for all $F \in \mathcal{F}_{h}^{\partial}$, since the exact solution $\boldsymbol{A}$ has a zero tangential trace on $\partial D$.

(2b) Bound on $\mathfrak{T}_{2}$. Reasoning as in the proof of Lemma 6.1, we infer that $\left|\mathfrak{T}_{2}\right| \leq c \xi_{\kappa_{r}}^{\frac{1}{2}} \max \left(1, \zeta_{\mu \kappa}^{\frac{1}{2}}\right)\left\|\boldsymbol{b}_{h}\right\|_{\boldsymbol{V}_{h}}$.

\subsection{Mollified error estimate}

We are going to assume in this section that there is a real number $r>0$ so that the exact solution $\boldsymbol{A}$ is such that $\kappa \nabla \times \boldsymbol{A} \in \boldsymbol{H}^{r}(D)$. Let $k \geq 1$ be the degree of the underlying finite elements. We define $p$ and $t$ as in Section 6.2 and we set $q=2$. Let us define the functional space

$$
\boldsymbol{V}_{\mathrm{S}}:=\left\{\boldsymbol{b} \in \boldsymbol{H}_{0}(\operatorname{curl} ; D) \mid \kappa \nabla \times \boldsymbol{b} \in \boldsymbol{L}^{p}(D), \nabla \times(\kappa \nabla \times \boldsymbol{b}) \in \boldsymbol{L}^{2}(D)\right\},
$$

Lemma 7.2 (Exact solution). If $\boldsymbol{A} \in \boldsymbol{H}_{0}(\operatorname{curl} ; D)$, with $\kappa \nabla \times \boldsymbol{A} \in \boldsymbol{H}^{r}(D), r>0$, then $\boldsymbol{A}$ is in $\boldsymbol{V}_{\mathrm{S}}$ as defined by (7.5).

Let us equip the space $\boldsymbol{V}_{\sharp}:=\boldsymbol{V}_{\mathrm{S}}+\boldsymbol{V}_{h}$ with the norm

$$
\|\boldsymbol{b}\|_{\boldsymbol{V}_{\sharp}}^{2}:=\|\boldsymbol{b}\|_{\boldsymbol{V}_{b}}^{2}+\sum_{K \in \overline{\mathcal{T}}_{h}^{\partial}} \kappa_{r, K}^{-1}\left(h_{K}^{d\left(\frac{1}{2}-\frac{1}{p}\right)}\|\kappa \nabla \times \boldsymbol{b}\|_{\boldsymbol{L}^{p}(K)}+h_{K}\|\nabla \times(\kappa \nabla \times \boldsymbol{b})\|_{\boldsymbol{L}^{2}(K)}\right)^{2},
$$

Compared with the norm defined by (7.4) used for the trimmed error estimate, we observe that there is now an additional term measuring $\kappa \nabla \times \boldsymbol{b}$ in the $\boldsymbol{L}^{p}$-norm, but the summation is now restricted to the smaller set $\overline{\mathcal{T}}_{h}^{\partial} \subsetneq \mathcal{T}_{h}^{\partial}$. Let us define the following sesquilinear form on $\boldsymbol{V}_{\sharp} \times \boldsymbol{V}_{h}$ :

$$
a_{\sharp}\left(\boldsymbol{v}, \boldsymbol{b}_{h}\right):=\int_{D}\left(\widetilde{\mu} \boldsymbol{v} \cdot \overline{\boldsymbol{b}}_{h}+\kappa \nabla \times \boldsymbol{v} \cdot \nabla \times \overline{\boldsymbol{b}}_{h}\right) \mathrm{d} x+\sum_{F \in \mathcal{F}_{h}^{\partial}}\left\langle\left((\kappa \nabla \times \boldsymbol{v})_{\mid K_{F}} \times \boldsymbol{n}\right)_{\mid F}, \Pi_{F}\left(\boldsymbol{b}_{h}\right)\right\rangle,
$$

where $\Pi_{F}$ is the $\ell^{2}$-orthogonal projection onto the hyperplane tangent to $F$, i.e., $\Pi_{F}\left(\boldsymbol{b}_{h}\right)=\boldsymbol{b}_{h}-\left(\boldsymbol{b}_{h}, \boldsymbol{n}\right)_{\ell^{2}} \boldsymbol{n}$. Notice that $\Pi_{F}\left(\boldsymbol{b}_{h}\right)$ is indeed a member of the space $\boldsymbol{Y}^{\mathrm{c}}(F)$ defined by $(5.14 \mathrm{~b})$ since $\Pi_{F}\left(\boldsymbol{b}_{h}\right) \cdot \boldsymbol{n}=0$, and that $\left\|\Pi_{F}\left(\boldsymbol{b}_{h}\right)\right\|_{\ell^{2}}=\left\|\boldsymbol{b}_{h} \times \boldsymbol{n}\right\|_{\ell^{2}}$.

Lemma 7.3 (Mollified error estimate). The assumptions of Lemma 4.8 hold true for the bilinear form $a_{\sharp}$ defined by (7.7) and the stabilization bilinear form $s_{h}$ defined by (3.11b). Moreover, the constant $\omega_{V_{\sharp}, W_{h}}^{\text {mol }}$ involved in (4.20) and the constant $\sigma_{V_{h}, W_{h}}$ involved in (4.22) are independent of the contrast in $\kappa$.

Proof. We only highlight the differences with respect to the proof of Lemma 6.3.

(1) Verification of (4.20). This is a direct consequence of (5.13), the Cauchy-Schwarz inequality, the choice (3.12) of the penalty parameter $\rho_{h}$, and the fact that $\left\|\rho_{h}^{\frac{1}{2}} \boldsymbol{w}_{h}\right\|_{L^{2}(\partial D)} \leq\left\|\boldsymbol{w}_{h}\right\|_{\boldsymbol{V}_{h}}$ for all $\boldsymbol{w}_{h} \in \boldsymbol{V}_{h}$.

(2) Proof of (4.21a). The argument is the same as in the proof of Lemma 6.3.

(3) Proof of (4.21b). Let $\mathcal{K}_{\delta}^{\mathrm{c}}: \boldsymbol{L}^{1}(D) \rightarrow \boldsymbol{C}^{\infty}(\bar{D})$ and $\mathcal{K}_{\delta}^{\mathrm{d}}: \boldsymbol{L}^{1}(D) \rightarrow C^{\infty}(\bar{D})$ be the mollification operators introduced in $\S 5.1$. The proof of $(4.21 \mathrm{~b})$ now relies on the following key commuting property:

$$
\nabla \times\left(\mathcal{K}_{\delta}^{\mathrm{c}}(\boldsymbol{\tau})\right)=\mathcal{K}_{\delta}^{\mathrm{d}}(\nabla \times \boldsymbol{\tau}),
$$

which holds true for all $\boldsymbol{\tau} \in \boldsymbol{L}^{1}(D)$ s.t. $\nabla \times \boldsymbol{\tau} \in \boldsymbol{L}^{1}(D)$. The rest of the argument follows the same lines as in the proof of Lemma 6.3.

(3) Verification of (4.22). The Cauchy-Schwarz inequality implies that $\left\|s_{h}\left(\boldsymbol{b}_{h}, \cdot\right)\right\|_{\boldsymbol{V}_{h}^{\prime}} \leq \eta_{0}\left\|\rho_{h}^{\frac{1}{2}} \boldsymbol{b}_{h}\right\|_{\boldsymbol{L}^{2}(\partial D)}$ for all $\boldsymbol{b}_{h} \in \boldsymbol{V}_{h}$, and (4.22) follows since any function $\boldsymbol{v}$ in $V=\boldsymbol{H}_{0}(\operatorname{curl} ; D)$ has a zero tangential trace on $\partial D$. 
Acknowledgement This material is based upon work supported in part by the National Science Foundation grants DMS 1620058, DMS 1619892, by the Air Force Office of Scientific Research, USAF, under grant/contract number FA99550-12-0358 and the Army Research Office, under grant number W911NF-151-0517. This work was done in part when the authors were visiting the Institut Henri Poincaré during the Fall 2016 Thematic Trimester "Numerical Methods for Partial Differential Equations". The support of IHP is gratefully acknowledged.

\section{References}

[1] C. Amrouche, C. Bernardi, M. Dauge, and V. Girault. Vector potentials in three-dimensional nonsmooth domains. Math. Methods Appl. Sci., 21(9):823-864, 1998.

[2] I. Babuška and A. K. Aziz. Survey lectures on the mathematical foundations of the finite element method. In The mathematical foundations of the finite element method with applications to partial differential equations (Proc. Sympos., Univ. Maryland, Baltimore, Md., 1972), pages 1-359. Academic Press, New York, 1972.

[3] S. Badia, R. Codina, T. Gudi, and J. Guzmán. Error analysis of discontinuous Galerkin methods for the Stokes problem under minimal regularity. IMA J. Numer. Anal., 34(2):800-819, 2014.

[4] L. Beirão da Veiga, F. Brezzi, A. Cangiani, G. Manzini, L. D. Marini, and A. Russo. Basic principles of virtual element methods. Math. Models Methods Appl. Sci., 23(1):199-214, 2013.

[5] C. Bernardi and F. Hecht. Error indicators for the mortar finite element discretization of the Laplace equation. Math. Comp., 71(240):1371-1403, 2002.

[6] A. Bonito, J.-L. Guermond, and F. Luddens. Regularity of the maxwell equations in heterogeneous media and lipschitz domains. J. Math. Anal. Appl., 408:498-512, 2013.

[7] D. Braess. Finite elements. Cambridge University Press, Cambridge, third edition, 2007. Theory, fast solvers, and applications in elasticity theory, Translated from the German by Larry L. Schumaker.

[8] S. C. Brenner and L. R. Scott. The mathematical theory of finite element methods, volume 15 of Texts in Applied Mathematics. Springer-Verlag, New York, second edition, 2002.

[9] H. Brezis. Functional analysis, Sobolev spaces and partial differential equations. Universitext. Springer, New York, 2011.

[10] C. Carstensen and M. Schedensack. Medius analysis and comparison results for first-order finite element methods in linear elasticity. IMA J. Numer. Anal., 35(4):1591-1621, 2015.

[11] J. Céa. Approximation variationnelle des problèmes aux limites. Ann. Inst. Fourier (Grenoble), 14: 345-444, 1964.

[12] D. A. Di Pietro and A. Ern. Mathematical Aspects of Discontinuous Galerkin Methods, volume 69 of Mathématiques \& Applications. Springer-Verlag, Berlin, 2012.

[13] A. Ern and J.-L. Guermond. Theory and Practice of Finite Elements, volume 159 of Applied Mathematical Sciences. Springer-Verlag, New York, NY, 2004.

[14] A. Ern and J.-L. Guermond. Discontinuous Galerkin methods for Friedrichs' systems. I. General theory. SIAM J. Numer. Anal., 44(2):753-778, 2006.

[15] A. Ern and J.-L. Guermond. Mollification in strongly Lipschitz domains with application to continuous and discrete de Rham complexes. Comput. Methods Appl. Math., 16(1):51-75, 2016.

[16] A. Ern and J.-L. Guermond. Finite element quasi-interpolation and best approximation. M2AN Math. Model. Numer. Anal., 51(4):1367-1385, 2017.

[17] K. O. Friedrichs. The identity of weak and strong extensions of differential operators. Trans. Amer. Math. Soc., 55:132-151, 1944. 
[18] T. Gudi. A new error analysis for discontinuous finite element methods for linear elliptic problems. Math. Comp., 79(272):2169-2189, 2010.

[19] S. Hofmann, M. Mitrea, and M. Taylor. Geometric and transformational properties of Lipschitz domains, Semmes-Kenig-Toro domains, and other classes of finite perimeter domains. J. Geom. Anal., 17(4):593-647, 2007.

[20] F. Jochmann. Regularity of weak solutions of Maxwell's equations with mixed boundary-conditions. Math. Methods Appl. Sci., 22(14):1255-1274, 1999.

[21] J. Leray. Sur le mouvement d'un liquide visqueux emplissant l'espace. Acta Math., 63(1):193-248, 1934.

[22] J. Nečas. Sur une méthode pour résoudre les équations aux dérivées partielles de type elliptique, voisine de la variationnelle. Ann. Scuola Norm. Sup. Pisa, 16:305-326, 1962.

[23] J. Nitsche. Über ein Variationsprinzip zur Lösung von Dirichlet-Problemen bei Verwendung von Teilräumen, die keinen Randbedingungen unterworfen sind. Abh. Math. Sem. Univ. Hamburg, 36: 9-15, 1971.

[24] J. Schöberl. Commuting quasi-interpolation operators for mixed finite elements. Technical Report ISC-01-10-MATH, Texas A\&M University, 2001. URL www.isc.tamu.edu/publications-reports/tr/0110.pdf.

[25] J. Schöberl. A posteriori error estimates for Maxwell equations. Math. Comp., 77(262):633-649, 2008.

[26] S. Sobolev. Sur un théorème d'analyse fonctionnelle. Rec. Math. [Mat. Sbornik] N.S., 4(46):471-497, 1938.

[27] G. Strang. Variational crimes in the finite element method. In A. Aziz, editor, The Mathematical Foundations of the Finite Element Method with Applications to Partial Differential Equations, New York, NY, 1972. Academic Press.

[28] A. Veeser and P. Zanotti. Private communication. 2016.

[29] R. Verfürth. A posteriori error estimation techniques for finite element methods. Numerical Mathematics and Scientific Computation. Oxford University Press, Oxford, 2013. 